\title{
EXISTENCE AND LARGE TIME STABILITY OF TRAVELING WAVE SOLUTIONS TO NONLINEAR BALANCE LAWS IN TRAFFIC FLOWS*
}

\author{
SHIH-WEI CHOU ${ }^{\dagger}$, JOHN M. HONG ${ }^{\ddagger}$, AND YING-CHIEH LIN§
}

\begin{abstract}
In this paper, we consider a nonlinear hyperbolic system of balance laws in Eulerian coordinates arising from a continuum traffic flow model whose source term consists of a relaxation and an extra term related to the non-uniform road widths. We establish the existence and large-time stability of traveling wave solutions for the initial value problem of such system. Contrast to previous results, there are four types of traveling waves according to the stability of the equilibria at $x= \pm \infty$. Under the entropy condition, the original and modified subcharacteristic conditions, together with a subsonic condition, we show by the weighted energy method that each type of traveling waves is asymptotically stable under small perturbations.
\end{abstract}

Key words. Conservation laws, nonlinear balance laws, traveling waves, stability, relaxation, weighted energy estimates, traffic flow.

AMS subject classifications. 5B20, 35B40, 35B45, 35L65, 76L05, 90B20.

\section{Introduction}

We consider the following nonlinear hyperbolic system of balance laws in Eulerian coordinates arising from the non-equilibrium traffic flow model

$$
\left\{\begin{array}{l}
(a \rho)_{t}+(a \rho u)_{x}=0, \\
u_{t}+u u_{x}+\frac{c^{2}(\rho)}{\rho} \rho_{x}=\frac{1}{\tau}\left(V_{*}(\rho)-u\right),
\end{array}\right.
$$

where $a=a(x, t)=a(x-s t)$ is a given traveling wave with speed $s$ related to the road width, $\rho, u$ are the density and velocity for the flow of the vehicle, $\frac{1}{\tau}\left(V_{*}(\rho)-u\right)$ is the relaxation term of (1.1) with relaxation time $\tau>0, V_{*}(\rho)$ is the equilibrium speed satisfying $V_{*}^{\prime}(\rho)<0$, and $c(\rho)$ is the traffic sound speed defined as

$$
c(\rho):=\rho V_{*}^{\prime}(\rho) .
$$

The system (1.1) is a simplified version of the full relaxation model

$$
\left\{\begin{array}{l}
(a \rho)_{t}+(a \rho u)_{x}=0, \\
u_{t}+u u_{x}+\frac{c^{2}(\rho)}{\rho} \rho_{x}=\frac{1}{\tau}\left(V_{*}(\rho)-u\right)-\frac{a_{t}}{a} c(\rho)-\frac{a_{x}}{a} c(\rho) V_{*}(\rho),
\end{array}\right.
$$

The first equation in (1.2) stands for the conservation of vehicle, while the second one describes drivers' acceleration behavior. In general, the equilibrium speed $V_{*}$ and the traffic sound speed $c$ may depend on $a_{x}, a_{t}$ [37]. However, since $a_{x}, a_{t}$ are very

*Received: July 18, 2012; accepted (in revised form): November 9, 2012. Communicated by Alberto Bressan.

${ }^{\dagger}$ Department of Mathematics, National Central University, Jhongli City 32001, Taiwan (swchou@ math.ncu.edu.tw).

${ }^{\ddagger}$ Department of Mathematics, National Central University, Jhongli City 32001, Taiwan (jhong@ math.ncu.edu.tw).

$\S$ Department of Mathematics, National Central University, Jhongli City 32001, Taiwan (linyj@ math.ncu.edu.tw). 
small in our consideration, their effects can be ignored in $V_{*}$ and $c$. Without loss of generality, we will demonstrate our results in the case

$$
V_{*}(\rho)=1-\rho,
$$

in which the maximal density and the free flow speed are normalized to 1 .

The model (1.1) simulates the traffic behavior near a particular vehicle or a moving barrier with low constant speed $s$, which blocks a single lane on a multi-lane highway. The speed $s>0(s<0$ respectively $)$ means that the barrier moves in the same (respectively opposite) direction with the other vehicles. The case $s=0$ expresses that the barrier does not move. In order to simplify the description of the physical meaning for model (1.1), we use the moving coordinate $z=x-s t$ with $z=0$ standing for the location of the barrier. In addition, we define $z>0(z<0$ respectively) as the front (back respectively) of the barrier. In this paper, we focus on the following two situations: (A) The other vehicles blocked by the barrier need to change lane when they arrive at a certain distance from the back of the barrier; see figure 1(a); (B) the other vehicles do not occupy the same lane with the barrier until they exceed the barrier by a certain distance in front of the barrier; see figure 1(b). We study how the traffic density changes with these two situations and when the density forms a traveling wave together with its stability. In this investigation, we always consider the traffic flow as a continuum fluid.

Let

$$
\tilde{\rho}:=a \rho, \quad m:=a \rho u=\tilde{\rho} u .
$$

Then, by (1.3), we can rewrite (1.1) as the following system:

$$
\left\{\begin{array}{l}
\tilde{\rho}_{t}+m_{x}=0, \\
m_{t}+g(a, \tilde{\rho}, m)_{x}=a_{x} P(a, \tilde{\rho})+\frac{1}{\tau}(f(a, \tilde{\rho})-m),
\end{array}\right.
$$

where

$$
\begin{aligned}
g(a, \tilde{\rho}, m) & =\frac{m^{2}}{\tilde{\rho}}+a P(a, \tilde{\rho}), \quad f(a, \tilde{\rho})=\tilde{\rho}\left(1-\frac{\tilde{\rho}}{a}\right), \\
P(a, \tilde{\rho}) & =\int^{\frac{\tilde{\rho}}{a}} c^{2}(s) d s=\frac{1}{3} \frac{\tilde{\rho}^{3}}{a^{3}} .
\end{aligned}
$$

Model (1.4) can be reformulated as a new relaxation system consisting of the relaxation term $\frac{1}{\tau}(f(a, \tilde{\rho})-m)$ with nonconvex equilibrium flux $f(a, \tilde{\rho})$, and the term $a_{x} P$ involving the traffic sound speed and the road width. In addition, compared to the previous models in $[15,17,18,19,21,22,24,25,38,41]$, the flux $g$ in (1.4) is more general due to the non-uniform road width and the non-constant traffic sound speed.

In this paper, we study the existence and nonlinear asymptotic stability of the traveling wave solutions $(a(z), U(z), V(z)), z=x-s t$, to the following initial value problem:

$$
\left\{\begin{array}{l}
\tilde{\rho}_{t}+m_{x}=0, \\
m_{t}+g(a, \tilde{\rho}, m)_{x}=a_{x} P(a, \tilde{\rho})+\frac{1}{\tau}(f(a, \tilde{\rho})-m), \\
(a, \tilde{\rho}, m)(x, 0)=\left(a, \tilde{\rho}_{0}, m_{0}\right)(x) \rightarrow\left(a_{ \pm}, \tilde{\rho}_{ \pm}, m_{ \pm}\right) \quad \text { as } x \rightarrow \pm \infty, \quad m_{ \pm}=f\left(a_{ \pm}, \tilde{\rho}_{ \pm}\right),
\end{array}\right.
$$


where $g, f, P$ are as in (1.5), and $a=a(z)$ is a monotone function satisfying the two-point boundary value problem

$$
\frac{d a}{d z}=R(a):=\kappa\left(a-a_{-}\right)\left(a-a_{+}\right), \quad a \rightarrow a_{ \pm} \quad \text { as } z \rightarrow \pm \infty .
$$

Here, $\kappa \in \mathbb{R}$ is an important parameter controlling the behavior of the traveling wave solutions. The equation (1.7) simulates the behavior of the drivers when they change lane behind (or in front of) the barrier. Since $\tilde{\rho}=a \rho$ and $0<\rho<1$ in our problem, it means that $\tilde{\rho}<a$. Throughout this paper, we consider the following two cases of $a, U$ at $z= \pm \infty$ :

$$
\begin{aligned}
& \text { (A) } \quad \kappa>0, \quad \tilde{\rho}_{-}<\tilde{\rho}_{+}<a_{+}<a_{-}, \\
& \text {(B) } \kappa<0, \quad \tilde{\rho}_{+}<\tilde{\rho}_{-}<a_{-}<a_{+} .
\end{aligned}
$$

With the speed $s$ given in system (1.1), the traveling waves $(a(z), U(z), V(z))$ together with their end states $\left(a_{ \pm}, \tilde{\rho}_{ \pm}, m_{ \pm}\right)$, where $m_{ \pm}$is given in (1.6), satisfy the RankineHugoniot condition

$$
-s\left(\tilde{\rho}_{+}-\tilde{\rho}_{-}\right)+\left(f\left(a_{+}, \tilde{\rho}_{+}\right)-f\left(a_{-}, \tilde{\rho}_{-}\right)\right)=0
$$

and the entropy condition

$$
Q(a(z), U(z))\left\{\begin{array}{ll}
>0 & \text { in case (A), } \\
<0 & \text { in case (B), }
\end{array} \quad \text { for all } z \in \mathbb{R},\right.
$$

where

$$
Q(a, \tilde{\rho}):=f(a, \tilde{\rho})-f\left(a_{ \pm}, \tilde{\rho}_{ \pm}\right)-s\left(\tilde{\rho}-\tilde{\rho}_{ \pm}\right) \quad \text { for } a, \tilde{\rho}>0 .
$$

Moreover, $(a(z), U(z), V(z))$ also satisfy the original sub-characteristic condition

$$
\lambda_{1}(a(z), U(z), V(z))<s<\lambda_{2}(a(z), U(z), V(z)),
$$

where

$$
\begin{aligned}
& \lambda_{1}(a(z), U(z), V(z))=\frac{1}{2}\left(g_{m}-\left(g_{m}^{2}+4 g_{\tilde{\rho}}\right)^{\frac{1}{2}}\right)(a(z), U(z), V(z)), \\
& \lambda_{2}(a(z), U(z), V(z))=\frac{1}{2}\left(g_{m}+\left(g_{m}^{2}+4 g_{\tilde{\rho}}\right)^{\frac{1}{2}}\right)(a(z), U(z), V(z)) .
\end{aligned}
$$

To obtain the nonlinear stability of the traveling wave profiles for our system, we focus on those traveling waves with $U(z)$ monotone. In addition, we impose two extra conditions on the traveling waves $(a(z), U(z), V(z))$ :

(subsonic)

$$
g_{\tilde{\rho}}>0 \text {, }
$$

(modified subcharacteristic) $\quad \frac{g_{m}-2 \sqrt{g_{\tilde{\rho}}}}{2}<f_{\tilde{\rho}}<\frac{g_{m}+2 \sqrt{g_{\tilde{\rho}}}}{2}$.

Note that, (1.11) is equivalent to $u^{2}<c^{2}(\rho)$. 


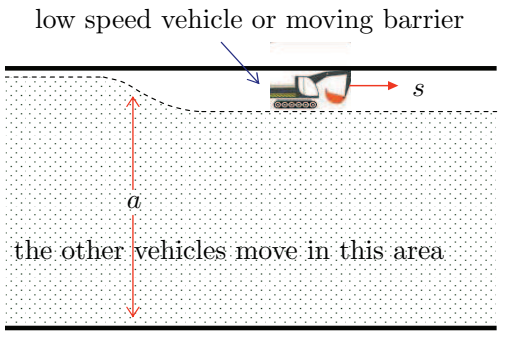

Figure 1(a). Case (A)

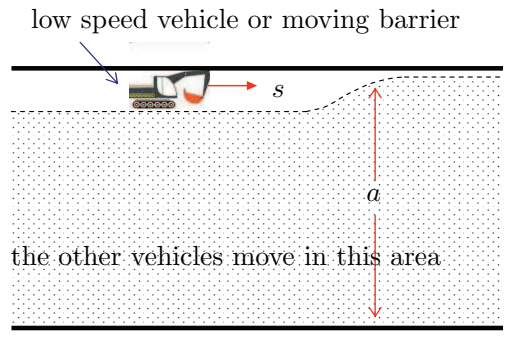

Figure 1(b). Case (B)

We recall the previous results related to this topic. The general hyperbolic conservation laws with relaxation were first studied by Liu [18] and later by Chen, Levermore, and Liu [1]. In [18, 21, 25], the subcharacteristic condition (1.10) was imposed for the existence and stability of traveling wave solutions. The asymptotic stability of traveling waves of scalar viscous conservation laws with nonconvex flux was studied by Matsumura and Nishihara [27], where a weighted energy estimate was invented for the stability results. When $a$ is a constant, i.e. the road is uniform, the model (1.1) was first derived by Zhang [40]. Such a model, with constant traffic sound speed in Lagrangian coordinates, can be transformed into

$$
\left\{\begin{array}{l}
v_{t}+u_{x}=0, \\
u_{t}+g(v)_{x}=\frac{1}{\tau}(f(v)-u) .
\end{array}\right.
$$

When $g$ is linear and $f$ is nonconvex in (1.13), the existence and stability of traveling waves was obtained by Liu, Wang, and Yang [25], and the results were extended to general $g$ by Li and Liu [21]. In [21, 25], the analogous weighted energy methods as in [27] were used for the stability results. The decay rate for the traveling waves of (1.13) was obtained by Li-Liu [21] and Liu-Woo-Yang [24]. We refer the readers to $[3,8,10,11,14,20,32,42]$ for more details of the existence and stability of solutions to relaxation systems and $[4,9,13,16,22,30,31,34]$ for the description of traffic flow models.

The above results give comprehensive contributions to the relaxation systems of traffic flows with uniform roads. However, these results cannot be applied to our system with non-uniform roads. Indeed, due to the appearance of $a$, the forms of flux and source in (1.4) become more complicated. Therefore, some difficulties arise to establish our results. First, unlike the results in [17, 21, 22, 25], the equation for the traveling wave $U$ is non-autonomous. Therefore, we augment the equation by adding (1.7) and consider the $2 \times 2$ system (2.11). This causes the variety of the stabilities near the equilibria $\left(a_{+}, \tilde{\rho}_{+}\right)$and $\left(a_{-}, \tilde{\rho}_{-}\right)$in $(2.11)$. Therefore, more techniques in dynamical systems such as the Central Manifold Theorem must applied. Second, to control the behaviors of traveling waves near the equilibria, we need to restrict the value of $\kappa$ in (1.7). Indeed, the suitable choices of $\kappa$ will lead to the monotonicity of $U$, which is necessary for the stability result. Finally, when we consider the stability of traveling wave solution $(a, U, V)(x-s t)$, if (3.1) fails, then the perturbed solution $(a, \tilde{\rho}, m)(x, t)$ will approach neither $(a, U, V)(x-s t)$ nor any of its shift $(a, U, V)(x+$ $\left.x_{0}-s t\right)$ as $t \rightarrow \infty$.

In our model (1.4), the traffic sound speed is not constant and the road width is not uniform. So, the flux $g$ also contains $m$ and $a$, and the equilibrium flux $f$ along the 
traveling wave solutions may not be concave in case (A) (or convex in case (B)) due to the appearance of $a$ in $f$. It follows that the traditional or weighted energy estimates, together with the original subcharacteristic condition in $[18,21,25]$, fail to provide the stability of the traveling wave solutions. To overcome the difficulty, we use the weighted energy estimates coupled with the subsonic and modified subcharacteristic conditions (1.11)-(1.12) to achieve the stability results.

We now give the notation of the weighted function space and state our main theorem. Given a weight function $w \geq 0$, we let $L_{w}^{2}$ denote the space of measurable functions $g$ satisfying $\sqrt{w} g \in L^{2}$ with norm

$$
\|g\|_{L_{w}^{2}}:=\left(\int w(x)|f(x)|^{2} d x\right)^{\frac{1}{2}} .
$$

Also, let $H_{w}^{j}, j>0$, denote the weighted Sobolev space with the norm

$$
\|g\|_{H_{w}^{j}}:=\left(\sum_{i=0}^{j}\left\|\partial_{x}^{i} g\right\|_{L_{w}^{2}}\right)^{\frac{1}{2}} .
$$

TheOREm 1.1. Consider the initial value problem (1.6), (1.7), where $\left|\tilde{\rho}_{+}-\tilde{\rho}_{-}\right|$is sufficiently small. Then, with the speed s given in system (1.1), there exist the traveling wave solutions $(a, U, V)(x-s t)$ together with their end states $\left(a_{ \pm}, \tilde{\rho}_{ \pm}, m_{ \pm}\right)$satisfying the conditions (1.8)-(1.10), such that $U(z)$ is monotone. Moreover,

(A1) if $\tilde{\rho}_{-}<\tilde{\rho}_{+}<a_{+}<a_{-}$fulfill (2.14) and $\kappa$ satisfies (2.20), then the profiles of $(a, U, V)(x-s t)$ are not unique and the orbits of $(a, U)$ are tangent to $(0,1)^{T}$ at the equilibria $\left(a_{+}, \tilde{\rho}_{+}\right)\left(\right.$sink) and $\left(a_{-}, \tilde{\rho}_{-}\right)$(source);

(A2) if $\tilde{\rho}_{-}<\tilde{\rho}_{+}<a_{+}<a_{-}$fulfill (2.15) and $\kappa$ satisfies (2.20), then the profile of $(a, U, V)(x-s t)$ is unique and the orbit of $(a, U)$ is tangent to $r_{1}\left(a_{+}, \tilde{\rho}_{+}\right)$at $\left(a_{+}, \tilde{\rho}_{+}\right)($saddle-node $)$and $(0,1)^{T}$ at $\left(a_{-}, \tilde{\rho}_{-}\right)$(source), where $r_{1}\left(a_{+}, \tilde{\rho}_{+}\right)$is as in $(2.13)$;

(B1) if $\tilde{\rho}_{+}<\tilde{\rho}_{-}<a_{-}<a_{+}$fulfill (2.16) and $\kappa$ satisfies (2.22), then the profile of $(a, U, V)(x-s t)$ is unique and the orbit of $(a, U)$ is tangent to $r_{1}\left(a_{+}, \tilde{\rho}_{+}\right)$at $\left(a_{+}, \tilde{\rho}_{+}\right)$(saddle) and $(0,1)^{T}$ at $\left(a_{-}, \tilde{\rho}_{-}\right)$(source), where $r_{1}\left(a_{+}, \tilde{\rho}_{+}\right)$is as in $(2.13)$;

(B2) if $\tilde{\rho}_{+}<\tilde{\rho}_{-}<a_{-}<a_{+}$fulfill (2.17) and $\kappa$ satisfies (2.22), then the profile of $(a, U, V)(x-s t)$ is unique and the orbit of $(a, U)$ is tangent to $r_{1}\left(a_{+}, \tilde{\rho}_{+}\right)$at $\left(a_{+}, \tilde{\rho}_{+}\right)($saddle $)$and $(0,1)^{T}$ at $\left(a_{-}, \tilde{\rho}_{-}\right)$(saddle-node), where $r_{1}\left(a_{+}, \tilde{\rho}_{+}\right)$is as in (2.13).

In addition, under the conditions (1.11) and (1.12), there exists a constant $\zeta>0$ such that if

$$
\left|a_{+}-a_{-}\right|+\left|\tilde{\rho}_{+}-\tilde{\rho}_{-}\right|+\left\|\left(\phi, \phi_{x}, \psi\right)(\cdot, 0)\right\|_{H^{2}}+\left\|\left(\phi, \phi_{x}, \psi\right)(\cdot, 0)\right\|_{L_{w}^{2}} \leq \zeta,
$$

where

$$
\begin{aligned}
(\phi, \psi)(x, 0)= & \left(\int_{-\infty}^{x}(\tilde{\rho}(y, 0)-U(y)) d y, m(x, 0)-V(x)\right), \\
& \int_{-\infty}^{\infty} \tilde{\rho}(x, 0) d x=\int_{-\infty}^{\infty} U(x) d x
\end{aligned}
$$


and the weight function $w$ is defined as

$$
w(a, U):=\left|\frac{\tau\left(U-\tilde{\rho}_{+}\right)\left(U-\tilde{\rho}_{-}\right)}{Q(a, U)+\tau a_{z}\left(P-g_{a}\right)}\right|,
$$

then the initial value problem (1.6), (1.7) has a unique global solution $(a, \tilde{\rho}, m)(x, t)$ satisfying

$$
\tilde{\rho}(x, t)-U(x-s t), m(x, t)-V(x-s t) \in C^{0}\left([0, \infty) ; H^{2} \cap L_{w}^{2}\right) \cap L^{2}\left(0, \infty ; H^{2} \cap L_{w}^{2}\right)
$$

and

$$
\sup _{x \in \mathbb{R}}|(\tilde{\rho}, m)(x, t)-(U, V)(x-s t)| \rightarrow 0 \quad \text { as } t \rightarrow \infty .
$$

Conditions (2.14)-(2.17) in Theorem 1.1 gives the mechanism to decide the magnitude of road-blocking $\left|a_{+}-a_{-}\right|$by the difference of the densities between the upstream and downstream of the car flow. Since the weights $w(a, U)$ in cases (A1), (A2), and (B1) are bounded, the space $L_{w}^{2}$ in the above theorem is equivalent to $L^{2}$. In the case (B2), $w(a, U)$ blows up as $z \rightarrow-\infty$, which will be explained in Section 3. So, we cannot remove the weight in this case.

The paper is organized as follows. In Section 2, we derive the subcharacteristic condition for system (1.1) and prove the existence of the desired traveling wave profiles to (1.6), (1.7) by the trapping region method. We classify the traveling wave solutions due to the stability of the equilibria at $z= \pm \infty$. In Section 3, we reformulate the stability problem by using the perturbations in $[3,17,19,21,24]$ to the underlying traveling wave solutions. In Section 4, the weighted energy estimates in [17, 19, 21, $24,29]$ are extended to our system with more general flux and source. The desired a priori estimates are obtained for the nonlinear stability of traveling wave profiles. Following the weighted energy method, we establish the nonlinear stability of traveling wave solutions for (1.6), (1.7) under conditions (1.8)-(1.12). In the last section, we give some conclusions of our results.

\section{Traveling wave solutions}

In this section, we prove the existence of traveling wave solutions to (1.6). We first derive the sub-characteristic condition of (1.4), which enables us to give the range of the traveling speed for the existence of traveling waves. To start, using the Chapman-Enskog expansion [18] to (1.4), we have the first order expansion

$$
\tilde{\rho}_{t}+f(a, \tilde{\rho})_{x}=0
$$

with the equilibrium characteristic $f_{\tilde{\rho}}(a, \tilde{\rho})$, and the second order expansion

$$
\tilde{\rho}_{t}+h\left(a, a_{x}, a_{t}, \tilde{\rho}, \tau\right)_{x}=\tau\left(\beta(a, \tilde{\rho}) \tilde{\rho}_{x}\right)_{x},
$$

where $h\left(a, a_{x}, a_{t}, \tilde{\rho}, \tau\right):=f(a, \tilde{\rho})-\tau\left(f_{a} a_{t}+g_{a} a_{x}+f_{a} a_{x}\left(g_{m}-f_{\tilde{\rho}}\right)-a_{x} P\right)$ and

$$
\beta(a, \tilde{\rho}):=g_{m} f_{\tilde{\rho}}-f_{\tilde{\rho}}^{2}+g_{\tilde{\rho}}
$$

On the other hand, system (1.4) can be written as

$$
\left\{\begin{array}{l}
\tilde{\rho}_{t}+m_{x}=0, \\
m_{t}+g_{\tilde{\rho}} \tilde{\rho}_{x}+g_{m} m_{x}=a_{x}\left(P-g_{a}\right)+\frac{1}{\tau}(f(a, \tilde{\rho})-m),
\end{array}\right.
$$


which means that (1.4) is strictly hyperbolic with characteristics $\lambda_{1}<\lambda_{2}$, where

$$
\lambda_{1}=\frac{1}{2}\left(g_{m}-\left(g_{m}^{2}+4 g_{\tilde{\rho}}\right)^{\frac{1}{2}}\right), \quad \lambda_{2}=\frac{1}{2}\left(g_{m}+\left(g_{m}^{2}+4 g_{\tilde{\rho}}\right)^{\frac{1}{2}}\right) .
$$

The derived equation (2.2) is well-posed only if the diffusion coefficient $\beta(a, \tilde{\rho})$ is positive. From (2.3) and (2.4), it follows that

$$
\beta(a, \tilde{\rho})=-\left(f_{\tilde{\rho}}(a, \tilde{\rho})-\lambda_{1}(a, \tilde{\rho}, f(a, \tilde{\rho}))\right)\left(f_{\tilde{\rho}}(a, \tilde{\rho})-\lambda_{2}(a, \tilde{\rho}, f(a, \tilde{\rho}))\right) .
$$

Thus, by the results of [18], the stability criterion becomes

$$
\lambda_{1}(a, \tilde{\rho}, f(a, \tilde{\rho}))<f_{\tilde{\rho}}(a, \tilde{\rho})<\lambda_{2}(a, \tilde{\rho}, f(a, \tilde{\rho})),
$$

which means that $f_{\tilde{\rho}}(a, \tilde{\rho})$ is sub-characteristic.

Next, letting $z=x-s t$, we now prove the existence of the traveling wave solutions

$$
(a, \tilde{\rho}, m)(x, t)=(a, U, V)(x-s t):=(a, U, V)(z)
$$

of (1.6) satisfying

$$
(a, U, V)(z) \rightarrow\left(a_{ \pm}, \tilde{\rho}_{ \pm}, m_{ \pm}\right) \quad \text { as } z \rightarrow \pm \infty, \quad m_{ \pm}=f\left(a_{ \pm}, \tilde{\rho}_{ \pm}\right) .
$$

In this paper, we look for the traveling wave solutions corresponding to the shock waves for (2.1). So, the traveling wave solutions together with speed $s$ and constant states $\tilde{\rho}_{ \pm}, a_{ \pm}$satisfy the Rankine-Hugoniot condition (1.8), the entropy condition (1.9), and the sub-characteristic condition (1.10). Note that the sub-characteristic condition (1.10) is based on (2.5), (2.14)-(2.17), and the assumption that $\tilde{\rho}_{-}, \tilde{\rho}_{+}$are sufficiently close. We look for the profiles of traveling wave solutions to (1.6) which satisfy (2.6) and the system

$$
\left\{\begin{array}{l}
-s U_{z}+V_{z}=0, \\
-s V_{z}+g(a, U, V)_{z}=\frac{1}{\tau}(f(a, U)-V)+a_{z} P(a, U),
\end{array}\right.
$$

where $g, f$, and $P$ are in (1.5). From the first equation of (2.7), $V_{z}=s U_{z}$. Plugging this into the second equation of $(2.7)$, we obtain

$$
-s^{2} U_{z}+g_{a} a_{z}+g_{\tilde{\rho}} U_{z}+s g_{m} U_{z}=\frac{1}{\tau}(f(a, U)-V)+a_{z} P .
$$

Integrating the first equation of (2.7) over $( \pm \infty, z)$ and using (2.6), we have

$$
-s U+V=-s \tilde{\rho}_{ \pm}+m_{ \pm}=-s \tilde{\rho}_{ \pm}+f\left(a_{ \pm}, \tilde{\rho}_{ \pm}\right) .
$$

Note that the above equality holds by the Rankine-Hugoniot condition. Combining (2.8) with (2.9), we arrive at

$$
U_{z}=\frac{Q(a, U)+\tau a_{z}\left(P-g_{a}\right)}{\tau\left(g_{\tilde{\rho}}+s g_{m}-s^{2}\right)},
$$

where

$$
\begin{aligned}
g_{a} & =g_{a}\left(a, U, s U-s \tilde{\rho}_{ \pm}+f\left(a_{ \pm}, \tilde{\rho}_{ \pm}\right)\right), \\
g_{\tilde{\rho}} & =g_{\tilde{\rho}}\left(a, U, s U-s \tilde{\rho}_{ \pm}+f\left(a_{ \pm}, \tilde{\rho}_{ \pm}\right)\right), \\
g_{m} & =g_{m}\left(a, U, s U-s \tilde{\rho}_{ \pm}+f\left(a_{ \pm}, \tilde{\rho}_{ \pm}\right)\right), \\
Q(a, U) & =f(a, U)-\left(s U-s \tilde{\rho}_{ \pm}+f\left(a_{ \pm}, \tilde{\rho}_{ \pm}\right)\right),
\end{aligned}
$$


and $V$ in (2.10) is replaced by the function of $U$ under (2.9). We observe that (2.10) is a non-autonomous equation due to the appearance of $a$, so the analysis in [21] cannot be applied directly. To overcome the difficulty, we augment (2.10) by adding the equation (1.7) for $a$. Then, we have the following equivalent $2 \times 2$ system:

$$
\left\{\begin{array}{l}
a_{z}=R(a)=\kappa\left(a-a_{-}\right)\left(a-a_{+}\right) \\
U_{z}=\frac{Q(a, U)+\tau R(a)\left(P-g_{a}\right)}{\tau\left(g_{\tilde{\rho}}+s g_{m}-s^{2}\right)}
\end{array}\right.
$$

where $Q$ is given in (1.9).

To prove the existence of solutions to (2.11), we use the Stable Manifold Theorem, the Center Manifold Theorem, and the method of trapping regions. To get the nonlinear stability of our traveling waves $(a(z), U(z), V(z))$, we tend to find those $U$ with monotonicity. We consider the following two cases of $a, U$ at $z= \pm \infty$ :

$$
\begin{aligned}
& \text { (A) } \kappa>0, \quad \tilde{\rho}_{-}<\tilde{\rho}_{+}<a_{+}<a_{-}, \\
& \text {(B) } \kappa<0, \quad \tilde{\rho}_{+}<\tilde{\rho}_{-}<a_{-}<a_{+} .
\end{aligned}
$$

Under (1.10), we have $g_{\tilde{\rho}}+s g_{m}-s^{2}>0$ along the traveling wave solutions. So, from the definitions of $Q$ and $R$, we can easily find that the system (2.11) has at least three equilibria including $\left(a_{+}, \tilde{\rho}_{+}\right)$and $\left(a_{-}, \tilde{\rho}_{-}\right)$in both cases (A) and (B). Moreover, all of the equilibria lie exactly on the intersection of the lines $a=a_{ \pm}$and the curve $Q(a, \tilde{\rho})=0$; see figures 2(a)-3(b). Our strategy is to find in each case a suitable trapping region whose boundary contains $\left(a_{+}, \tilde{\rho}_{+}\right),\left(a_{-}, \tilde{\rho}_{-}\right)$. Also, the other equilibria lie outside the closure of the region.

Now, we study the stability of $\left(a_{+}, \tilde{\rho}_{+}\right)$and $\left(a_{-}, \tilde{\rho}_{-}\right)$. Let $\Lambda$ denote the variational matrix of (2.11). Then the eigenvalues of $\Lambda$ are

$$
\begin{aligned}
& \nu_{1}(a, \tilde{\rho})=\kappa\left(2 a-a_{+}-a_{-}\right), \\
& \nu_{2}(a, \tilde{\rho})=\frac{\partial_{\tilde{\rho}}\left(Q+\tau R\left(P-g_{a}\right)\right)\left(g_{\tilde{\rho}}+s g_{m}-s^{2}\right)-\left(Q+\tau R\left(P-g_{a}\right)\right) \partial_{\tilde{\rho}}\left(g_{\tilde{\rho}}+s g_{m}-s^{2}\right)}{\tau\left(g_{\tilde{\rho}}+s g_{m}-s^{2}\right)^{2}} .
\end{aligned}
$$

It follows that the eigenvalues of $\left.\Lambda\right|_{\left(a_{+}, \tilde{\rho}_{+}\right)}$and $\left.\Lambda\right|_{\left(a_{-}, \tilde{\rho}_{-}\right)}$are respectively

$$
\begin{aligned}
& \left\{\nu_{1}\left(a_{+}, \tilde{\rho}_{+}\right)=\kappa\left(a_{+}-a_{-}\right), \quad \nu_{2}\left(a_{+}, \tilde{\rho}_{+}\right)=\frac{Q_{\tilde{\rho}}\left(a_{+}, \tilde{\rho}_{+}\right)}{\tau\left(g_{\tilde{\rho}}+s g_{m}-s^{2}\right)}\right\}, \\
& \left\{\nu_{1}\left(a_{-}, \tilde{\rho}_{-}\right)=\kappa\left(a_{-}-a_{+}\right), \quad \nu_{2}\left(a_{-}, \tilde{\rho}_{-}\right)=\frac{Q_{\tilde{\rho}}\left(a_{-}, \tilde{\rho}_{-}\right)}{\tau\left(g_{\tilde{\rho}}+s g_{m}-s^{2}\right)}\right\},
\end{aligned}
$$

where

$$
\begin{aligned}
& Q_{\tilde{\rho}}\left(a_{+}, \tilde{\rho}_{+}\right)=\frac{1}{a_{+}\left(\tilde{\rho}_{+}-\tilde{\rho}_{-}\right)}\left(2 \tilde{\rho}_{+} \tilde{\rho}_{-}-\tilde{\rho}_{+}^{2}-\frac{a_{+}}{a_{-}} \tilde{\rho}_{-}^{2}\right), \\
& Q_{\tilde{\rho}}\left(a_{-}, \tilde{\rho}_{-}\right)=\frac{1}{a_{-}\left(\tilde{\rho}_{+}-\tilde{\rho}_{-}\right)}\left(\frac{a_{-}}{a_{+}} \tilde{\rho}_{+}^{2}-2 \tilde{\rho}_{+} \tilde{\rho}_{-}+\tilde{\rho}_{-}^{2}\right) .
\end{aligned}
$$

The corresponding right eigenvectors at $\left(a_{-}, \tilde{\rho}_{-}\right)$and $\left(a_{+}, \tilde{\rho}_{+}\right)$are respectively

$$
r_{1}\left(a_{-}, \tilde{\rho}_{-}\right)=\left(\begin{array}{c}
1 \\
\frac{Q_{a}+\tau \kappa\left(a_{-}-a_{+}\right)\left(P-g_{a}\right)}{\tau\left(\nu_{1}-\nu_{2}\right)\left(g_{\tilde{\rho}}+s g_{m}-s^{2}\right)}
\end{array}\right), \quad r_{2}\left(a_{-}, \tilde{\rho}_{-}\right)=\left(\begin{array}{l}
0 \\
1
\end{array}\right),
$$




$$
r_{1}\left(a_{+}, \tilde{\rho}_{+}\right)=\left(\begin{array}{c}
1 \\
\frac{Q_{a}+\tau \kappa\left(a_{+}-a_{-}\right)\left(P-g_{a}\right)}{\tau\left(\nu_{1}-\nu_{2}\right)\left(g_{\tilde{\rho}}+s g_{m}-s^{2}\right)}
\end{array}\right), \quad r_{2}\left(a_{+}, \tilde{\rho}_{+}\right)=\left(\begin{array}{l}
0 \\
1
\end{array}\right) .
$$

According to the stability of $\left(a_{+}, \tilde{\rho}_{+}\right)$and $\left(a_{-}, \tilde{\rho}_{-}\right)$, we further divide cases (A), (B) into the following subcases:

(A1) If $\kappa>0, \tilde{\rho}_{-}<\tilde{\rho}_{+}<a_{+}<a_{-}$and

$$
\frac{a_{-}-a_{+}}{a_{-}}<\left(\frac{\tilde{\rho}_{+}-\tilde{\rho}_{-}}{\tilde{\rho}_{-}}\right)^{2},
$$

then $Q_{\tilde{\rho}}\left(a_{+}, \tilde{\rho}_{+}\right)<0$ and $Q_{\tilde{\rho}}\left(a_{-}, \tilde{\rho}_{-}\right)>0$, which implies that $\left(a_{-}, \tilde{\rho}_{-}\right)$is a source and $\left(a_{+}, \tilde{\rho}_{+}\right)$is a sink.

(A2) If $\kappa>0, \tilde{\rho}_{-}<\tilde{\rho}_{+}<a_{+}<a_{-}$and

$$
\frac{a_{-}-a_{+}}{a_{-}}=\left(\frac{\tilde{\rho}_{+}-\tilde{\rho}_{-}}{\tilde{\rho}_{-}}\right)^{2},
$$

then $Q_{\tilde{\rho}}\left(a_{+}, \tilde{\rho}_{+}\right)=0$ and $Q_{\tilde{\rho}}\left(a_{-}, \tilde{\rho}_{-}\right)>0$, which implies that $\left(a_{-}, \tilde{\rho}_{-}\right)$is a source and $\left(a_{+}, \tilde{\rho}_{+}\right)$is a saddle-node.

(B1) If $\kappa<0, \tilde{\rho}_{+}<\tilde{\rho}_{-}<a_{-}<a_{+}$and

$$
\left(\frac{\tilde{\rho}_{-}-\tilde{\rho}_{+}}{\tilde{\rho}_{+}}\right)^{2}<\frac{a_{+}-a_{-}}{a_{+}} \leq C\left(\frac{\tilde{\rho}_{-}-\tilde{\rho}_{+}}{\tilde{\rho}_{+}}\right)^{2}
$$

where $C>1$ is a constant, then $Q_{\tilde{\rho}}\left(a_{+}, \tilde{\rho}_{+}\right)>0$ and $Q_{\tilde{\rho}}\left(a_{-}, \tilde{\rho}_{-}\right)>0$, which implies that $\left(a_{-}, \tilde{\rho}_{-}\right)$is a source and $\left(a_{+}, \tilde{\rho}_{+}\right)$is a saddle.

(B2) If $\kappa<0, \tilde{\rho}_{+}<\tilde{\rho}_{-}<a_{-}<a_{+}$and

$$
\frac{a_{+}-a_{-}}{a_{+}}=\left(\frac{\tilde{\rho}_{-}-\tilde{\rho}_{+}}{\tilde{\rho}_{+}}\right)^{2},
$$

then $Q_{\tilde{\rho}}\left(a_{+}, \tilde{\rho}_{+}\right)>0$ and $Q_{\tilde{\rho}}\left(a_{-}, \tilde{\rho}_{-}\right)=0$, which implies that $\left(a_{-}, \tilde{\rho}_{-}\right)$is a saddle-node and $\left(a_{+}, \tilde{\rho}_{+}\right)$is a saddle.

There are still two cases,

$$
\frac{a_{-}-a_{+}}{a_{-}}>\left(\frac{\tilde{\rho}_{+}-\tilde{\rho}_{-}}{\tilde{\rho}_{-}}\right)^{2} \quad \text { in case }(\mathrm{A})
$$

and

$$
\frac{a_{+}-a_{-}}{a_{+}}<\left(\frac{\tilde{\rho}_{-}-\tilde{\rho}_{+}}{\tilde{\rho}_{+}}\right)^{2} \quad \text { in case }(\mathrm{B})
$$

which are not included in our list. However, after proving the existence of the desired solutions for (A1)-(B2), we will show that these two cases can be ruled out under the entropy condition (1.9).

Next, we find a trapping region in each subcase and prove the existence of those solutions $(a(z), U(z))$ with $U$ monotone.

For case (A1), we define

$$
\theta:=\max \left\{\frac{Q_{\tilde{\rho}}\left(a_{+}, \tilde{\rho}_{+}\right)}{\tau\left(g_{\tilde{\rho}}+s g_{m}-s^{2}\right)\left(a_{+}-a_{-}\right)}, \frac{Q_{\tilde{\rho}}\left(a_{-}, \tilde{\rho}_{-}\right)}{\tau\left(g_{\tilde{\rho}}+s g_{m}-s^{2}\right)\left(a_{-}-a_{+}\right)}\right\},
$$


and choose the parameter $\kappa$ in (1.7) satisfying

$$
\theta<\kappa<\alpha \theta,
$$

where $\alpha>1$ is a constant. It follows that

$$
0>\nu_{2}\left(a_{+}, \tilde{\rho}_{+}\right)>\nu_{1}\left(a_{+}, \tilde{\rho}_{+}\right) \quad \text { and } \quad \nu_{1}\left(a_{-}, \tilde{\rho}_{-}\right)>\nu_{2}\left(a_{-}, \tilde{\rho}_{-}\right)>0 .
$$

By the Stable Manifold Theorem, if the solutions exist, then their orbits are tangent to $r_{2}\left(a_{+}, \tilde{\rho}_{+}\right)$at $\left(a_{+}, \tilde{\rho}_{+}\right)$and $r_{2}\left(a_{-}, \tilde{\rho}_{-}\right)$at $\left(a_{-}, \tilde{\rho}_{-}\right)$.

We now find a trapping region for case (A1). Let $D_{1}$ be the region enclosed by the curves $\left\{\Gamma_{1}^{i}\right\}_{i=1}^{5}$ where

(1) $\Gamma_{1}^{1}$ is the line segment connecting $\left(a_{+}, \tilde{\rho}_{+}\right)$and $\left(a_{-}, \tilde{\rho}_{+}\right)$;

(2) $\Gamma_{1}^{2}$ is the positive orbit of the initial value problem

$$
\left\{\begin{array}{l}
a_{z}=R(a), \\
\tilde{\rho}_{z}=\frac{Q(a, \tilde{\rho})}{\tau\left(g_{\tilde{\rho}}+s g_{m}-s^{2}\right)}, \\
a(0)=a_{*}, \quad \tilde{\rho}(0)=\tilde{\rho}_{*},
\end{array}\right.
$$

where $a_{*}>a_{+}, \tilde{\rho}_{*}<\tilde{\rho}_{+}$,

$$
g_{\tilde{\rho}}=g_{\tilde{\rho}}\left(a, \tilde{\rho}, s \tilde{\rho}-s \tilde{\rho}_{ \pm}+f\left(a_{ \pm}, \tilde{\rho}_{ \pm}\right)\right), \quad g_{m}=g_{m}\left(a, \tilde{\rho}, s \tilde{\rho}-s \tilde{\rho}_{ \pm}+f\left(a_{ \pm}, \tilde{\rho}_{ \pm}\right)\right),
$$

and $\left(a_{*}, \tilde{\rho}_{*}\right)$ is sufficiently close to $\left(a_{+}, \tilde{\rho}_{+}\right)$;

(3) $\Gamma_{1}^{3}$ is the line segment connecting $\left(a_{*}, \tilde{\rho}_{*}\right)$ and $\left(a_{*}, \tilde{\rho}_{* *}\right)$ such that $\tilde{\rho}_{* *}<\tilde{\rho}_{*}$ and $Q\left(a_{*}, \tilde{\rho}_{* *}\right)=0$

(4) $\Gamma_{1}^{4}$ is the portion of curve satisfying $Q(a, \tilde{\rho})=0$ between $\left(a_{*}, \tilde{\rho}_{* *}\right)$ and $\left(a_{-}, \tilde{\rho}_{-}\right)$;

(5) $\Gamma_{1}^{5}$ is the line segment connecting $\left(a_{-}, \tilde{\rho}_{+}\right)$and $\left(a_{-}, \tilde{\rho}_{-}\right)$; see figure $2(\mathrm{a})$.

We notice that, by the existence and uniqueness theorem of ODEs and the fact that $\left(a_{+}, \tilde{\rho}_{+}\right)$is a sink of $(2.21)$, the solution $(a, \tilde{\rho})$ exists for $z \geq 0$ with $\lim _{z \rightarrow+\infty}(a, \tilde{\rho})=$ $\left(a_{+}, \tilde{\rho}_{+}\right)$. Therefore, $\Gamma_{1}^{2}$ is well-defined with left-end point $\left(a_{+}, \tilde{\rho}_{+}\right)$. It implies that $D_{1}$ is a closed region.

Let $\vec{n}$ denote the unit outer normal to the boundary of our trapping region and

$$
F(a, \tilde{\rho}):=\left(R(a), \frac{Q(a, \tilde{\rho})+\tau R(a)\left(P-g_{a}\right)}{\tau\left(g_{\tilde{\rho}}+s g_{m}-s^{2}\right)}\right)^{T}
$$

be the vector field of (2.11). To prove the existence of the solutions, it is sufficient to show that $D_{1}$ is a negative invariant region, which means that $\left.\vec{n} \cdot F\right|_{\Gamma_{1}^{i}} \geq 0$ for $i=1, \cdots, 5$. The calculation of $\left.\vec{n} \cdot F\right|_{\Gamma_{1}^{i}}$ is given as follows:

(1) On $\Gamma_{1}^{1}$,

$$
\begin{aligned}
\left.\vec{n} \cdot F\right|_{\Gamma_{1}^{1}} & =(0,1) \cdot\left(R, \frac{Q+\tau R\left(P-g_{a}\right)}{\tau\left(g_{\tilde{\rho}}+s g_{m}-s^{2}\right)}\right)=\frac{Q+\tau R\left(P-g_{a}\right)}{\tau\left(g_{\tilde{\rho}}+s g_{m}-s^{2}\right)} \\
& =\frac{a-a_{+}}{\tau\left(g_{\tilde{\rho}}+s g_{m}-s^{2}\right)}\left(\frac{\tilde{\rho}_{+}^{2}}{a a_{+}}-\tau \kappa a\left(a-a_{-}\right) P_{a}\left(a, \tilde{\rho}_{+}\right)\right) .
\end{aligned}
$$

In this case, we have 


$$
0>Q_{\tilde{\rho}}\left(a_{+}, \tilde{\rho}_{+}\right)=-\frac{1}{a_{+}}\left(\tilde{\rho}_{+}-\tilde{\rho}_{-}\right)+\frac{\tilde{\rho}_{-}^{2}}{a_{+} a_{-}}\left\{\frac{a_{-}-a_{+}}{\tilde{\rho}_{+}-\tilde{\rho}_{-}}\right\}>-\frac{1}{a_{+}}\left(\tilde{\rho}_{+}-\tilde{\rho}_{-}\right)
$$

and

$$
0<Q_{\tilde{\rho}}\left(a_{-}, \tilde{\rho}_{-}\right)=\frac{1}{a_{-}}\left(\tilde{\rho}_{+}-\tilde{\rho}_{-}\right)+\frac{\tilde{\rho}_{+}^{2}}{a_{+} a_{-}}\left\{\frac{a_{-}-a_{+}}{\tilde{\rho}_{+}-\tilde{\rho}_{-}}\right\}<\left\{\frac{1}{a_{-}}+\frac{\tilde{\rho}_{+}^{2}}{a_{+} \tilde{\rho}_{-}^{2}}\right\}\left(\tilde{\rho}_{+}-\tilde{\rho}_{-}\right) .
$$

Thus, by the choice of $\kappa(2.20)$ and above inequalities, we obtain

$$
\tau \kappa a\left(a-a_{-}\right) P_{a}\left(a, \tilde{\rho}_{+}\right)<\frac{\tilde{\rho}_{+}^{2}}{a a_{+}}
$$

if $\left|\tilde{\rho}_{+}-\tilde{\rho}_{-}\right|$is sufficiently small. Hence $\left.\vec{n} \cdot F\right|_{\Gamma_{1}^{1}}>0$.

(2) On $\Gamma_{1}^{2}$, in view of (2.21) and by $\left.P_{a}\right|_{\Gamma_{1}^{2}}<0,\left.Q\right|_{\Gamma_{1}^{2}}>0$, we have

$$
\begin{aligned}
\left.\vec{n} \cdot F\right|_{\Gamma_{1}^{2}} & =\left(-1, \frac{d a}{d \tilde{\rho}}\right) \cdot\left(R, \frac{Q+\tau R\left(P-g_{a}\right)}{\tau\left(g_{\tilde{\rho}}+s g_{m}-s^{2}\right)}\right) \\
& =\frac{\left(Q+\tau R\left(P-g_{a}\right)\right) R}{Q}-R=\left.\frac{-\tau a P_{a} R^{2}}{Q}\right|_{\Gamma_{1}^{2}}>0 .
\end{aligned}
$$

(3) On $\Gamma_{1}^{3}$, we have $\left.R\right|_{\Gamma_{1}^{3}}<0$, which leads to

$$
\left.\vec{n} \cdot F\right|_{\Gamma_{1}^{3}}=(-1,0) \cdot\left(R, \frac{Q+\tau R\left(P-g_{a}\right)}{\tau\left(g_{\tilde{\rho}}+s g_{m}-s^{2}\right)}\right)=-\left.R\right|_{\Gamma_{1}^{3}}>0
$$

(4) On $\Gamma_{1}^{4}$, since $R<0, P_{a}<0$ and $\frac{d a}{d \tilde{\rho}}<0$ on $\Gamma_{1}^{4}$, we have

$$
\begin{aligned}
\left.\vec{n} \cdot F\right|_{\Gamma_{1}^{4}} & =\left(-1, \frac{d a}{d \tilde{\rho}}\right) \cdot\left(R, \frac{Q+\tau R\left(P-g_{a}\right)}{\tau\left(g_{\tilde{\rho}}+s g_{m}-s^{2}\right)}\right) \\
& =-R\left(1+\frac{d a}{d \tilde{\rho}} \frac{a P_{a}}{g_{\tilde{\rho}}+s g_{m}-s^{2}}\right)>0 .
\end{aligned}
$$

(5) On $\Gamma_{1}^{5}$, we have

$$
\left.\vec{n} \cdot F\right|_{\Gamma_{1}^{5}}=(1,0) \cdot\left(R, \frac{Q+\tau R\left(P-g_{a}\right)}{\tau\left(g_{\tilde{\rho}}+s g_{m}-s^{2}\right)}\right)=\left.R\right|_{\Gamma_{1}^{5}}=0 .
$$

We then show the existence of the solutions of (2.11) for case (A1).

For case (A2), choose the parameter $\kappa$ as given in (2.20). We have

$$
0=\nu_{2}\left(a_{+}, \tilde{\rho}_{+}\right)>\nu_{1}\left(a_{+}, \tilde{\rho}_{+}\right) \quad \text { and } \quad \nu_{1}\left(a_{-}, \tilde{\rho}_{-}\right)>\nu_{2}\left(a_{-}, \tilde{\rho}_{-}\right)>0 .
$$




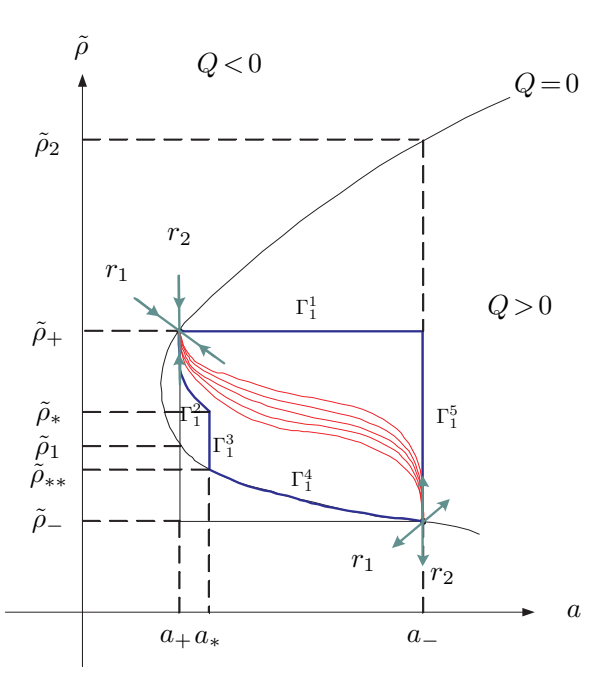

Figure 2(a). Case (A1)

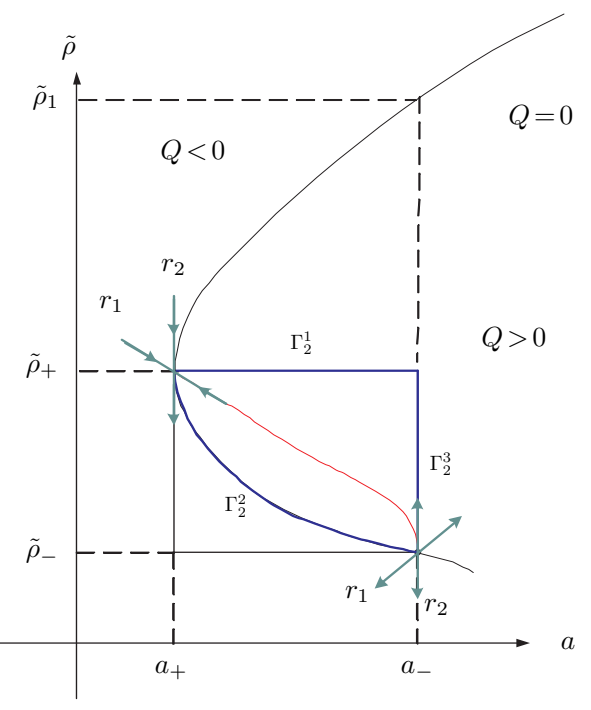

Figure 2(b). Case (A2)

Then, by the Center Manifold Theorem, there exists a unique 1-dimensional stable manifold $W^{s}\left(a_{+}, \tilde{\rho}_{+}\right)$tangent to $r_{1}\left(a_{+}, \tilde{\rho}_{+}\right)$at $\left(a_{+}, \tilde{\rho}_{+}\right)$, and the analytic center manifold $W^{c}\left(a_{+}, \tilde{\rho}_{+}\right)$of $\left(a_{+}, \tilde{\rho}_{+}\right)$is exactly the line $a=a_{+}$. In addition, the Stable Manifold Theorem states that if the solutions exist, then their orbits are tangent to $r_{2}\left(a_{-}, \tilde{\rho}_{-}\right)$at $\left(a_{-}, \tilde{\rho}_{-}\right)$.

To describe the trapping region for case (A2), we let $D_{2}$ be the region enclosed by the curves $\left\{\Gamma_{2}^{i}\right\}_{i=1}^{3}$, where

(1) $\Gamma_{2}^{1}$ is the line segment connecting $\left(a_{+}, \tilde{\rho}_{+}\right)$and $\left(a_{-}, \tilde{\rho}_{+}\right)$;

(2) $\Gamma_{2}^{2}$ is the portion of curve satisfying $Q(a, \tilde{\rho})=0$ between $\left(a_{+}, \tilde{\rho}_{+}\right)$and $\left(a_{-}, \tilde{\rho}_{-}\right)$;

(3) $\Gamma_{2}^{3}$ is the line segment connecting $\left(a_{-}, \tilde{\rho}_{+}\right)$and $\left(a_{-}, \tilde{\rho}_{-}\right)$; see figure $2(\mathrm{~b})$.

A similar analysis to case (A1) gives that $D_{2}$ is a negative invariant region, which implies the existence of the solutions. Note that $D_{2}$ contains the stable manifold $W^{s}\left(a_{+}, \tilde{\rho}_{+}\right)$in the zone $\left\{a_{+}<a<a_{-}\right\}$and leaves the analytic center manifold $W^{c}\left(a_{+}, \tilde{\rho}_{+}\right)$outside its closure, except for the equilibrium $\left(a_{+}, \tilde{\rho}_{+}\right)$. We see that all center manifolds of $\left(a_{+}, \tilde{\rho}_{+}\right)$must be tangent to $W^{c}\left(a_{+}, \tilde{\rho}_{+}\right)$at $\left(a_{+}, \tilde{\rho}_{+}\right)$. So, a solution $(a(z), U(z))$ along any center manifold of $\left(a_{+}, \tilde{\rho}_{+}\right)$will eventually leave our trapping region as $z \rightarrow \infty$. Thus, the solutions in our trapping region $D_{2}$ are tangent to $r_{2}\left(a_{-}, \tilde{\rho}_{-}\right)$at $\left(a_{-}, \tilde{\rho}_{-}\right)$as $z \rightarrow-\infty$ and then along the stable manifold $W^{s}\left(a_{+}, \tilde{\rho}_{+}\right)$ until $z=\infty$; moreover, the orbit of these traveling waves is unique.

Next, we turn to case (B1). Define

$$
\theta^{\prime}:=\frac{Q_{\tilde{\rho}}\left(a_{-}, \tilde{\rho}_{-}\right)}{\tau\left(g_{\tilde{\rho}}+s g_{m}-s^{2}\right)\left(a_{-}-a_{+}\right)},
$$

and choose the parameter $\kappa$ in (1.7) satisfying

$$
\alpha^{\prime} \theta^{\prime}<\kappa<\theta^{\prime}
$$


where $\alpha^{\prime}>1$ is a constant. It follows that

$$
\nu_{2}\left(a_{+}, \tilde{\rho}_{+}\right)>0>\nu_{1}\left(a_{+}, \tilde{\rho}_{+}\right) \quad \text { and } \quad \nu_{1}\left(a_{-}, \tilde{\rho}_{-}\right)>\nu_{2}\left(a_{-}, \tilde{\rho}_{-}\right)>0 .
$$

Then, by the Stable Manifold Theorem, if the solutions exist, then the orbits are tangent to $r_{1}\left(a_{+}, \tilde{\rho}_{+}\right)$at $\left(a_{+}, \tilde{\rho}_{+}\right)$and $r_{2}\left(a_{-}, \tilde{\rho}_{-}\right)$at $\left(a_{-}, \tilde{\rho}_{-}\right)$.

We define the region $D_{3}$ enclosed by the curves $\left\{\Gamma_{3}^{i}\right\}_{i=1}^{3}$, where

(1) $\Gamma_{3}^{1}$ is the portion of curve satisfying $Q(a, \tilde{\rho})=0$ between $\left(a_{+}, \tilde{\rho}_{+}\right),\left(a_{-}, \tilde{\rho}_{-}\right)$;

(2) $\Gamma_{3}^{2}$ is the line segment connecting $\left(a_{-}, \tilde{\rho}_{-}\right)$and $\left(a_{-}, \tilde{\rho}_{+}\right)$;

(3) $\Gamma_{3}^{3}$ is the line segment connecting $\left(a_{-}, \tilde{\rho}_{+}\right)$and $\left(a_{+}, \tilde{\rho}_{+}\right)$; see figure $3(\mathrm{a})$.

Following an analogous analysis as in case (A1), we calculate the follows terms.

(1) On $\Gamma_{3}^{1}$, following the facts that $\left.\frac{d a}{d \tilde{\rho}}\right|_{\Gamma_{3}^{1}}<0,\left.P_{a}\right|_{\Gamma_{3}^{1}}<0$, we have

$$
\begin{aligned}
\left.\vec{n} \cdot F\right|_{\Gamma_{3}^{1}} & =\left(1,-\frac{d a}{d \tilde{\rho}}\right) \cdot\left(R, \frac{Q+\tau R\left(P-g_{a}\right)}{\tau\left(g_{\tilde{\rho}}+s g_{m}-s^{2}\right)}\right) \\
& =R\left(1+\frac{d a}{d \tilde{\rho}} \frac{a P_{a}}{g_{\tilde{\rho}}+s g_{m}-s^{2}}\right)>0 .
\end{aligned}
$$

(2) On $\Gamma_{3}^{2}$, we have $\left.\vec{n} \cdot F\right|_{\Gamma_{3}^{2}}=\left.R\right|_{\Gamma_{3}^{2}}=0$.

(3) On $\Gamma_{3}^{3}$, we obtain

$$
\begin{aligned}
\left.\vec{n} \cdot F\right|_{\Gamma_{3}^{3}} & =(0,-1) \cdot\left(R, \frac{Q+\tau R\left(P-g_{a}\right)}{\tau\left(g_{\tilde{\rho}}+s g_{m}-s^{2}\right)}\right) \\
& =\frac{a_{+}-a}{\tau\left(g_{\tilde{\rho}}+s g_{m}-s^{2}\right)}\left(\frac{\tilde{\rho}_{+}^{2}}{a a_{+}}-\tau \kappa a\left(a-a_{-}\right) P_{a}\left(a, \tilde{\rho}_{+}\right)\right) .
\end{aligned}
$$

In this case,

$$
\begin{aligned}
0<Q_{\tilde{\rho}}\left(a_{-}, \tilde{\rho}_{-}\right) & =\frac{1}{a_{-}\left(\tilde{\rho}_{+}-\tilde{\rho}_{-}\right)}\left(\frac{a_{-}}{a_{+}} \tilde{\rho}_{+}^{2}-2 \tilde{\rho}_{+} \tilde{\rho}_{-}+\tilde{\rho}_{-}^{2}\right) \\
& =\frac{1}{a_{-}}\left(\tilde{\rho}_{+}-\tilde{\rho}_{-}\right)+\frac{\tilde{\rho}_{+}^{2}}{a_{+} a_{-}}\left\{\frac{a_{-}-a_{+}}{\tilde{\rho}_{+}-\tilde{\rho}_{-}}\right\}<\left\{\frac{1}{a_{-}}+\frac{C \tilde{\rho}_{+}^{2}}{a_{+} \tilde{\rho}_{-}^{2}}\right\}\left(\tilde{\rho}_{+}-\tilde{\rho}_{-}\right) .
\end{aligned}
$$

Thus, by the choice of $\kappa(2.22)$ and above inequalities, we obtain

$$
\tau \kappa a\left(a-a_{-}\right) P_{a}\left(a, \tilde{\rho}_{+}\right)<\frac{\tilde{\rho}_{+}^{2}}{a a_{+}}
$$

if $\left|\tilde{\rho}_{+}-\tilde{\rho}_{-}\right|$is sufficiently small. Hence $\left.\vec{n} \cdot F\right|_{\Gamma_{3}^{3}}>0$. Since $\left(a_{+}, \tilde{\rho}_{+}\right)$in this case is a saddle, by the above analysis we have the existence and uniqueness of the solution up to a shift in case (B1).

In case (B2), choose $\kappa$ as in (2.22). Then we obtain

$$
\nu_{2}\left(a_{+}, \tilde{\rho}_{+}\right)>0>\nu_{1}\left(a_{+}, \tilde{\rho}_{+}\right) \quad \text { and } \quad \nu_{1}\left(a_{-}, \tilde{\rho}_{-}\right)>\nu_{2}\left(a_{-}, \tilde{\rho}_{-}\right)=0 .
$$

The Center Manifold Theorem implies that there exists a unique 1-dimensional unstable manifold $W^{u}\left(a_{-}, \tilde{\rho}_{-}\right)$tangent to $r_{1}\left(a_{-}, \tilde{\rho}_{-}\right)$at $\left(a_{-}, \tilde{\rho}_{-}\right)$and the analytic center manifold $W^{c}\left(a_{-}, \tilde{\rho}_{-}\right)$of $\left(a_{-}, \tilde{\rho}_{-}\right)$is exactly $a=a_{-}$. The Stable Manifold Theorem says that if the solutions exist, then the orbit is unique and tangent to $r_{1}\left(a_{+}, \tilde{\rho}_{+}\right)$at 
$\left(a_{+}, \tilde{\rho}_{+}\right)$. The definition of the trapping region $D_{4}$ for case (B2) is just the same as that of $D_{3}$ in case (B1); see figure 3(b). Applying a similar argument to case (B1), we have that $D_{4}$ is a negative invariant region, which leads to the existence of the solutions. The closure of the trapping region $D_{4}$ contains the analytic center manifold $W^{c}\left(a_{-}, \tilde{\rho}_{-}\right)$in the zone $\left\{\tilde{\rho}_{+} \leq \tilde{\rho} \leq \tilde{\rho}_{-}\right\}$and leaves the unstable manifold $W^{u}\left(a_{-}, \tilde{\rho}_{-}\right)$ outside except for the equilibrium $\left(a_{-}, \tilde{\rho}_{-}\right)$. Hence the orbit of the solutions is tangent to $r_{2}\left(a_{-}, \tilde{\rho}_{-}\right)$at $\left(a_{-}, \tilde{\rho}_{-}\right)$.

We now claim that, under the entropy condition (1.9), the states satisfying (2.18) or (2.19) do not exist. To show this, by previous analysis, the solutions $(a(z), U(z))$ satisfy either $\tilde{\rho}_{-}<U(z)<\tilde{\rho}_{+}$or $\tilde{\rho}_{+}<U(z)<\tilde{\rho}_{-}$. In case (A), (1.9) implies

$$
\frac{f(a, U)-f\left(a_{+}, \tilde{\rho}_{+}\right)}{U-\tilde{\rho}_{+}}-s<0 .
$$

Considering $a$ as a function of $U$ and taking $z \rightarrow \infty$, we obtain

$$
\begin{aligned}
0 & \geq \lim _{z \rightarrow \infty} \frac{f(a, U)-f\left(a_{+}, \tilde{\rho}_{+}\right)}{U-\tilde{\rho}_{+}}-s \\
& =\lim _{z \rightarrow \infty} \frac{f(a, U)-f\left(a, \tilde{\rho}_{+}\right)+f\left(a, \tilde{\rho}_{+}\right)-f\left(a_{+}, \tilde{\rho}_{+}\right)}{U-\tilde{\rho}_{+}}-s \\
& =f_{\tilde{\rho}}\left(a_{+}, \tilde{\rho}_{+}\right)+f_{a}\left(a_{+}, \tilde{\rho}_{+}\right) \frac{d a}{d U}-s=Q_{\tilde{\rho}}\left(a_{+}, \tilde{\rho}_{+}\right) .
\end{aligned}
$$

Then, (2.12) implies the desired result for case (A). The argument for case (B) is similar.

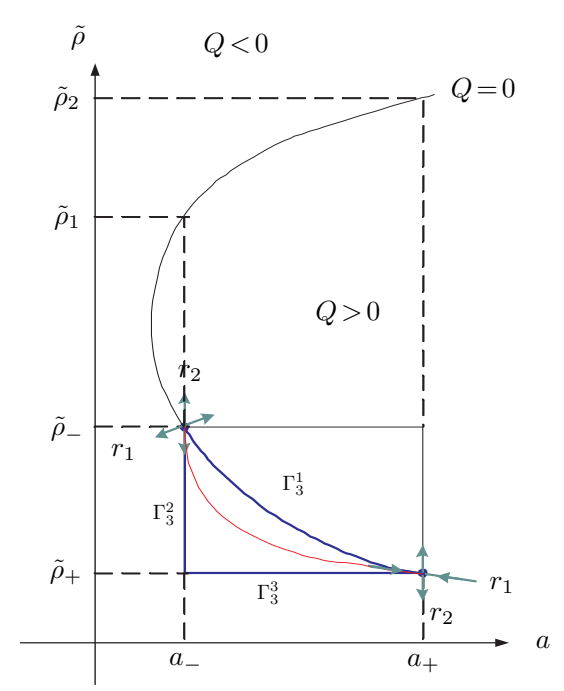

Figure 3(a). Case (B1)

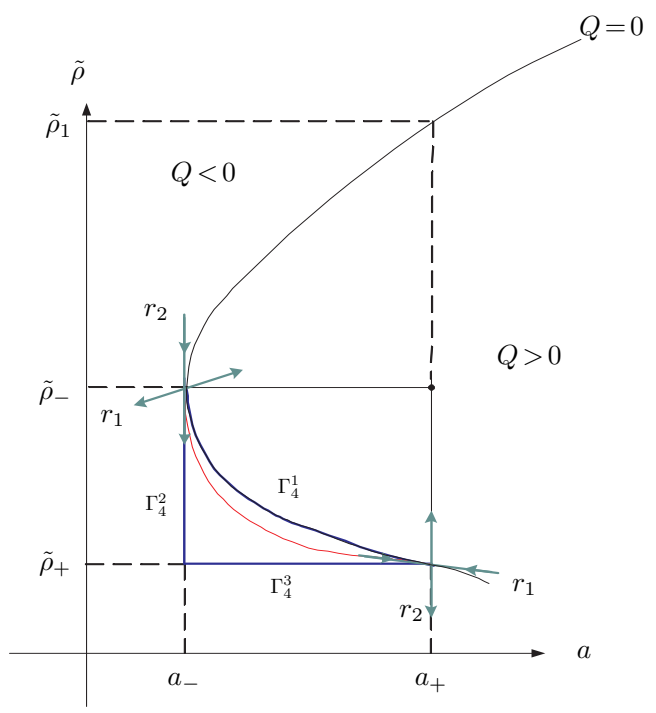

Figure 3(b). Case (B2)

We have the following lemma for the existence of the desired traveling waves to (1.6), (1.7).

Lemma 2.1. Consider the initial value problem (1.6), (1.7), where $\left|\tilde{\rho}_{+}-\tilde{\rho}_{-}\right|$is suffciently small and $\left(a_{ \pm}, \tilde{\rho}_{ \pm}, m_{ \pm}\right)$is as in (1.6). Let the speed $s$ be given in (1.1). 
(A1) If $\tilde{\rho}_{-}<\tilde{\rho}_{+}<a_{+}<a_{-}$fulfill (2.14) and $\kappa$ satisfies (2.20), then there exist traveling wave solutions $(a, U, V)(x-s t)$ satisfying the conditions (1.8)-(1.10) such that $\tilde{\rho}_{-}<U<\tilde{\rho}_{+}$. The orbits of $(a, U)(x-s t)$ are tangent to $r_{2}\left(a_{+}, \tilde{\rho}_{+}\right)$ at $\left(a_{+}, \tilde{\rho}_{+}\right)(\operatorname{sink})$, and $r_{2}\left(a_{-}, \tilde{\rho}_{-}\right)$at $\left(a_{-}, \tilde{\rho}_{-}\right)$(source).

(A2) If $\tilde{\rho}_{-}<\tilde{\rho}_{+}<a_{+}<a_{-}$fulfill (2.15) and $\kappa$ satisfies (2.20), then there exists a unique profile of traveling wave solution $(a, U, V)(x-s t)$ satisfying the conditions (1.8)-(1.10) such that $\tilde{\rho}_{-}<U<\tilde{\rho}_{+}$. The orbit of $(a, U)$ is tangent to $r_{1}\left(a_{+}, \tilde{\rho}_{+}\right)$at $\left(a_{+}, \tilde{\rho}_{+}\right)($saddle-node $)$and $r_{2}\left(a_{-}, \tilde{\rho}_{-}\right)$at $\left(a_{-}, \tilde{\rho}_{-}\right)$(source).

(B1) If $\tilde{\rho}_{+}<\tilde{\rho}_{-}<a_{-}<a_{+}$fulfill (2.16) and $\kappa$ satisfies (2.22), then there exists a unique profile of traveling wave solution $(a, U, V)(x-s t)$ satisfying the conditions (1.8)-(1.10) such that $\tilde{\rho}_{+}<U<\tilde{\rho}_{-}$. The orbit of $(a, U)(x-s t)$ is tangent to $r_{1}\left(a_{+}, \tilde{\rho}_{+}\right)$at $\left(a_{+}, \tilde{\rho}_{+}\right)($saddle $)$, and $r_{2}\left(a_{-}, \tilde{\rho}_{-}\right)$at $\left(a_{-}, \tilde{\rho}_{-}\right)$(source).

(B2) If $\tilde{\rho}_{+}<\tilde{\rho}_{-}<a_{-}<a_{+}$fulfill (2.17) and $\kappa$ satisfies (2.22), then there exists a unique profile of traveling wave solution $(a, U, V)(x-s t)$ satisfying the conditions (1.8)-(1.10) such that $\tilde{\rho}_{+}<U<\tilde{\rho}_{-}$. The orbit of $(a, U)(x-s t)$ is tangent to $r_{1}\left(a_{+}, \tilde{\rho}_{+}\right)$at $\left(a_{+}, \tilde{\rho}_{+}\right)$(saddle), and $r_{2}\left(a_{-}, \tilde{\rho}_{-}\right)$at $\left(a_{-}, \tilde{\rho}_{-}\right)$(saddle-node).

We emphasize that, in contrast to the other cases in Lemma 2.1, the traveling waves of (1.6), (1.7) in case (A1) may not be unique even up to the shifts of profiles. To see this, let $W^{s}\left(a_{+}, \tilde{\rho}_{+}\right), W^{u}\left(a_{-}, \tilde{\rho}_{-}\right)$be respectively the stable manifold of $\left(a_{+}, \tilde{\rho}_{+}\right)$ and the unstable manifold of $\left(a_{-}, \tilde{\rho}_{-}\right)$. Then, we have

$$
\operatorname{dim}\left(W^{s}\left(a_{+}, \tilde{\rho}_{+}\right)\right)+\operatorname{dim}\left(W^{u}\left(a_{-}, \tilde{\rho}_{-}\right)\right)-\operatorname{dim}\left(\mathbb{R}^{2}\right)=2>1,
$$

which implies the multiplicity of the traveling wave profiles.

In the end of this section, we prove that $U(z)$ in our traveling wave solutions to each case of Lemma 2.1 is strictly monotone, which is given in the following lemma.

Lemma 2.2. Let $(a(z), U(z), V(z))$ be a traveling wave solution of (1.6), (1.7) given in Lemma 2.1. Then, $U(z)$ is strictly monotone. Furthermore, $\left|U_{z}\right| \geq C\left|a_{z}\right|$ for all $z \in \mathbb{R}$ and some constant $C>0$.

Proof. We give the proof for case (A1) of Lemma 2.1; the proofs for the other cases are similar. In this case, we want to prove that $U(z)$ is strictly increasing. Let $\Sigma_{1}$ be the portion of $\{(a, \tilde{\rho}): Q(a, \tilde{\rho})=0\}$ between $\left(a_{+}, \tilde{\rho}_{1}\right)$ and $\left(a_{-}, \tilde{\rho}_{-}\right)$, where $\left(a_{+}, \tilde{\rho}_{1}\right)$ is the equilibrium outside $D$; see figure 1 . It is easy to verify that $Q_{a}>0$ on $\Sigma_{1}$. To see $Q_{\tilde{\rho}}>0$ on $\Sigma_{1}$, we let

$$
\Sigma_{0}:=\left\{(a, \tilde{\rho}): Q_{\tilde{\rho}}(a, \tilde{\rho})=0\right\} .
$$

Also let $\left(a_{0}, \tilde{\rho}_{0}\right)$ denote the point in $\{Q=0\}$ such that $a_{0}:=\min \{a: Q(a, \tilde{\rho})=0\}$. By a direct calculation, $\Sigma_{0}$ is the line passing through the origin and $\left(a_{0}, \tilde{\rho}_{0}\right)$. Then, from the definition of $\Sigma_{1}$, we observe that $\operatorname{dist}\left(\Sigma_{0}, \Sigma_{1}\right)>0$ and $\Sigma_{1}$ lies on the region of $\left\{Q_{\tilde{\rho}}>0\right\}$. Hence, $\left.Q_{\tilde{\rho}}\right|_{\Sigma_{1}} \geq C_{1}$ for some constant $C_{1}>0$. It follows that there exists a constant $C_{2}<0$ such that

$$
\left.\frac{d \tilde{\rho}}{d a}\right|_{\Sigma_{1}}=-\left.\frac{Q_{a}}{Q_{\tilde{\rho}}}\right|_{\Sigma_{1}} \leq C_{2}<0
$$

which implies that $\tilde{\rho}$ on $\Sigma_{1}$ is a strictly decreasing function of $a$. Next, let $\Sigma_{2}$ be the portion of $\left\{(a, \tilde{\rho}): Q(a, \tilde{\rho})+\tau R(a)\left(P-g_{a}\right)=0\right\}$ between $\left(a_{+}, \tilde{\rho}_{1}\right)$ and $\left(a_{-}, \tilde{\rho}_{-}\right)$. Following a similar analysis, for such $C_{2}$, we can find sufficiently small $\left|a_{+}-a_{-}\right|$such that $\tilde{\rho}$ on $\Sigma_{2}$ is also a strictly decreasing function of $a$. 
If $U(z)$ is not strictly increasing, then there exists a point $z_{0} \in \mathbb{R}$ such that $U_{z}\left(z_{0}\right)=$ 0 . It follows that $\left(a\left(z_{0}\right), U\left(z_{0}\right)\right) \in \Sigma_{2}$ and $F\left(a\left(z_{0}\right), U\left(z_{0}\right)\right)$ is transversal to $\Sigma_{2}$, since $\tilde{\rho}$ on $\Sigma_{2}$ is also a strictly decreasing function of $a$. So, the orbit of $(a(z), U(z)), z>z_{0}$ lies on the left of $\Sigma_{2}$, in which the second component of $F$ is negative. Therefore, by the fact that $\Sigma_{1}$ is decreasing, we have $F(a(z), U(z)), z>z_{0}$, is also transversal to $\Sigma_{1}$, which means that $(a(z), U(z))$ leaves the trapping region $D$ for large $z$. We then have the contradiction.

We have shown that $\left|\frac{U_{z}}{a_{z}}\right|>0$ for all $z \in \mathbb{R}$. By Lemma 2.1 and the properties of eigenvectors $r_{1}$ and $r_{2}$ at the end states $\left(a_{ \pm}, \tilde{\rho}_{ \pm}\right)$, we have $\left|\frac{U_{z}}{a_{z}}\right| \geq C$ as $z \rightarrow \pm \infty$ for some constant $C>0$, which implies that $\left|\frac{U_{z}}{a_{z}}\right| \geq C$ for all $z \in \mathbb{R}$.

\section{Reformulation of the stability problem}

From the results of Section 2, we are able to find the traveling wave profiles of problem (1.6), (1.7). To clarify the stability problem, some comments are in order. For any fixed $a(x-s t)$, and given end states $\left(a_{ \pm}, \tilde{\rho}_{ \pm}, m_{ \pm}\right)$, Lemma 2.1 states that, in cases (A2), (B1), and (B2), the problem admits only one profile of traveling wave solution with $U$ monotone, while in case (A1) there exist multiple profiles of traveling wave solutions. We can easily find from figure $2(\mathrm{a})$ that two different profiles of solutions $\left(a, U_{1}, V_{1}\right)(x-s t),\left(a, U_{2}, V_{2}\right)(x-s t)$ in (A1) correspond to two different initial total masses; more precisely,

$$
\int_{-\infty}^{\infty}\left(U_{1}(x)-U_{2}(x)\right) d x \neq 0
$$

Now, with a fixed road width condition $a(x-s t)$ and given a traveling wave solution $(a, U, V)(x-s t)$ of (1.6) in Lemma 2.1, we reformulate the stability problems of $(a, U, V)(x-s t)$ for all cases (A1)-(B2), which will be given as follows:

Suppose $(a, \tilde{\rho}, m)(x, t)$ is a perturbed solution of the traveling wave $(a, U, V)(x-$ $s t)$. Then the solution $(a, \tilde{\rho}, m)(x, t)$ exists globally and approaches the traveling wave solution $(a, U, V)(x-s t)$ as $t \rightarrow \infty$ if the difference of the total mass is zero, that is,

$$
\int_{-\infty}^{\infty}(\tilde{\rho}(x, 0)-U(x)) d x=0
$$

Otherwise, if (3.1) fails, then the perturbed solution $(a, \tilde{\rho}, m)(x, t)$ will approach neither $(a, U, V)(x-s t)$ nor any of its shift $(a, U, V)\left(x+x_{0}-s t\right)$ as $t \rightarrow \infty$. In this situation, $(a, \tilde{\rho}, m)(x, t)$ of case (A1) will approach another traveling wave profile $\left(a, U_{1}, V_{1}\right)(x-s t)$ such that

$$
\int_{-\infty}^{\infty}\left(\tilde{\rho}(x, 0)-U_{1}(x)\right) d x=0
$$

if the perturbation is small enough. Moreover, we are not able to predict the behavior of $(a, \tilde{\rho}, m)(x, t)$ in the other cases if (3.1) fails.

The stability is obtained based on the weighted energy estimate, which can be considered as the extension of the results in [17, 21, 25]. We look for the perturbed solution in the following form:

$$
(a, \tilde{\rho}, m)(x, t)=(a, U, V)(z)+\left(0, \phi_{z}, \psi\right)(z, t),
$$

where $z=x-s t$. Since the traveling wave solution $(a, U, V)(x-s t)$ satisfies $(3.1)$, the first equation of (1.6) implies that

$$
\phi( \pm \infty, t)=\int_{-\infty}^{\infty}(\tilde{\rho}(x, t)-U(x-s t)) d x=\int_{-\infty}^{\infty}(\tilde{\rho}(x, 0)-U(x)) d x=0
$$


To start, we substitute (3.2) into (1.6). In view of (2.7), and after integrating the first equation with respect to $z$, we obtain that the perturbation $(\phi, \psi)$ satisfies

$$
\left\{\begin{aligned}
& -s \phi_{z}+\phi_{t}+\psi=0 \\
& -s \psi_{z}+\psi_{t}+\left(g\left(a, U+\phi_{z}, V+\psi\right)-g(a, U, V)\right)_{z} \\
= & a_{z}\left(P\left(a, U+\phi_{z}\right)-P(a, U)\right)+\frac{1}{\tau}\left(f\left(a, U+\phi_{z}\right)-f(a, U)-\psi\right) .
\end{aligned}\right.
$$

The first equation of (3.3) gives

$$
\psi=s \phi_{z}-\phi_{t}
$$

Substituting (3.4) into the second equation of (3.3), we obtain the equation for $\phi$ given by

$$
\begin{aligned}
L(a, \phi) & :=\left(\phi_{t}-s \phi_{z}\right)_{t}-s\left(\phi_{t}-s \phi_{z}\right)_{z}-\left(g_{\tilde{\rho}} \phi_{z}+g_{m}\left(s \phi_{z}-\phi_{t}\right)\right)_{z}+\frac{1}{\tau} \phi_{t}+\mu \phi_{z} \\
& =-F\left(a, V, U, \phi_{z}, \phi_{t}\right) .
\end{aligned}
$$

Here,

$$
\mu:=\frac{1}{\tau} Q_{\tilde{\rho}}(a, U)+a_{z} P_{\tilde{\rho}}(a, U)=\frac{1}{\tau}\left(f_{\tilde{\rho}}(a, U)-s\right)+a_{z} P_{\tilde{\rho}}(a, U),
$$

$Q$ is in (1.9), and

$$
F\left(a, V, U, \phi_{z}, \phi_{t}\right):=F_{1}+F_{2}
$$

where

$$
\begin{aligned}
F_{1}:=- & \left\{g\left(a, U+\phi_{z}, V+\psi\right)-g(a, U, V)-g_{\tilde{\rho}}(a, U, V) \phi_{z}-g_{m}(a, U, V) \psi\right\}_{z}, \\
F_{2}:=\frac{1}{\tau}( & \left.f\left(a, U+\phi_{z}\right)-f(a, U)-f_{\tilde{\rho}}(a, U) \phi_{z}\right) \\
& +a_{z}\left(P\left(a, U+\phi_{z}\right)-P(a, U)-P_{\tilde{\rho}}(a, U) \phi_{z}\right) .
\end{aligned}
$$

We notice that $L$ is more complicated than the ones in $[17,21,25]$ due to the form of the flux, relaxation, and the appearance of $a$. The corresponding initial data of (3.5) become

$$
\phi(z, 0):=\phi_{0}(z), \quad \phi_{t}(z, 0)=s \phi_{0}^{\prime}(z)-\psi(z, 0):=\phi_{1}(z) .
$$

Then, the asymptotic stability of $(U, V)$ indicates that the perturbation $\left(\phi_{z}, \psi\right)$ decays to zero as $t \rightarrow \infty$.

To carry out our results, we give the weight function $w(a, U)$ in (1.14). Note that the behavior of the traveling wave solutions is closely related to the properties of $w$ and the function spaces involved with $w$. By the results in Section 2, it is obvious that $w>0$ for all traveling wave solutions of (1.6). Furthermore, for any given traveling wave solution $(a, U, V)(z)$ in all of our cases (A1)-(B2), since

$$
\lim _{z \rightarrow+\infty}\left|\frac{U(z)-\tilde{\rho}_{+}}{a(z)-a_{+}}\right| \geq C>0 \text { and } \lim _{z \rightarrow-\infty}\left|\frac{U(z)-\tilde{\rho}_{-}}{a(z)-a_{-}}\right| \geq C>0,
$$

we have $w(a(z), U(z)) \geq C$ as $z \rightarrow \pm \infty$ for some constant $C>0$. This implies that $w$ is bounded below by a positive constant and hence $L_{w}^{2} \subset L^{2}, H_{w}^{j} \subset H^{j}$ for $j>0$. On the 
other hand, we investigate whether $w$ goes to infinity as $z \rightarrow \pm \infty$. For cases (A1), (A2), and (B1), since the orbits of $(a, U)(z)$ are not tangent to the curve $\{(a, \tilde{\rho}): Q(a, \tilde{\rho})=0\}$ at the two end points $\left(a_{ \pm}, \tilde{\rho}_{ \pm}\right), w$ does not blow up as $z \rightarrow \pm \infty$ and hence we obtain that the weighted spaces $L_{w}^{2}$ and $H_{w}^{j}, j>0$, are equivalent to the usual spaces $L^{2}$ and $H^{j}$ respectively. However, in case (B2) we find that the orbit of $(a, U)(z)$ is tangent to $\{Q=0\}$ at $\left(a_{-}, \tilde{\rho}_{-}\right)$. Also we observe that $Q_{\tilde{\rho}}\left(a_{-}, \tilde{\rho}_{-}\right)=0$ under the condition (2.18) and

$$
\lim _{z \rightarrow-\infty} \frac{a(z)-a_{-}}{U(z)-\tilde{\rho}_{-}}=0 .
$$

It follows from the mean value theorem that $w$ blows up as $z \rightarrow-\infty$. Thus, we cannot replace the weighted spaces by the unweighted versions. For the convenience of presenting our stability results, we preserve the weighted form of the function spaces for all cases.

Now we introduce the solution space of the problem (3.5), (3.8) as follows:

$$
\begin{gathered}
X(0, T):=\left\{\phi(z, t): \phi \in C^{0}\left([0, T) ; H^{3} \cap H_{w}^{1}\right) \cap C^{1}\left([0, T) ; H^{2} \cap L_{w}^{2}\right),\right. \\
\left.\phi_{z}, \phi_{t} \in L^{2}\left(0, T ; H^{2} \cap L_{w}^{2}\right)\right\},
\end{gathered}
$$

with $0<T \leq \infty$. In view of $(3.5)$, we have

$$
\psi(z, t) \in C^{0}\left([0, T) ; H^{2} \cap L_{w}^{2}\right) \cap L^{2}\left(0, T ; H^{2} \cap L_{w}^{2}\right) .
$$

Therefore, by the Sobolev embedding theorem, if we let

$$
\mathcal{E}(t)=\sup _{0 \leq s \leq t}\left\{\|\phi(s)\|_{H^{3}}+\left\|\phi_{t}(s)\right\|_{H^{2}}+\|\phi(s)\|_{H_{w}^{1}}+\left\|\phi_{t}(s)\right\|_{L_{w}^{2}}\right\},
$$

then

$$
\sup _{z \in \mathbb{R}}\left\{|\phi|,\left|\phi_{z}\right|,\left|\phi_{z z}\right|,\left|\phi_{t}\right|,\left|\phi_{z t}\right|\right\} \leq C \mathcal{E}(t) .
$$

Thus, Theorem 1.1 is a consequence of the following theorem.

TheOREM 3.1. Under the same hypothesis given in Theorem 1.1, there exists a constant $\varepsilon_{1}>0$ such that if $\mathcal{E}(0) \leq \varepsilon_{1}$, then the problem (3.5), (3.8) has a unique global solution $\phi \in X(0, \infty)$ satisfying

$$
\mathcal{E}^{2}(t)+\int_{0}^{t}\left\|\left(\phi_{t}, \phi_{z}\right)\right\|_{H^{2}}^{2} d s+\int_{0}^{t}\left\|\left(\phi_{t}, \phi_{z}\right)\right\|_{L_{w}^{2}}^{2} d s \leq C \mathcal{E}^{2}(0)
$$

for $t \in[0, \infty)$. Furthermore,

$$
\sup _{z \in \mathbb{R}}\left|\left(\phi_{t}, \phi_{z}\right)\right| \rightarrow 0 \quad \text { as } t \rightarrow \infty .
$$

We notice that, if the perturbation $\phi$ fulfills Theorem 3.1, then by $(3.4),(\phi, \psi)$ becomes a global solution of $(3.3)$ with $(\phi, \psi)(z, 0)=\left(\phi_{0}, s \phi_{0}^{\prime}-\phi_{1}\right)(z)$. Therefore, following the existence of the traveling wave, we have the desired global solution of problem (1.6) through the relation (3.2). In addition, the solution of (1.6) is unique in $C^{0}\left(0, T ; H^{2} \cap L_{w}^{2}\right)$. Therefore, Theorem 1.1 follows from Theorem 3.1. Furthermore, by virtue of $L$ in (3.5), and following (3.10), we have

$$
\left\|\left(\phi_{t}, \phi_{z}\right)\right\|_{H^{1}} \rightarrow 0 \quad \text { as } t \rightarrow \infty
$$


and it follows that

$$
\begin{aligned}
\phi_{t}^{2}+\phi_{z}^{2} & =\int_{\infty}^{z}\left(2 \phi_{t} \phi_{t z}+2 \phi_{z} \phi_{z z}\right)(y, t) d y \\
& \leq 2\left(\int_{-\infty}^{\infty}\left(\phi_{t}^{2}+\phi_{z}^{2}\right) d y\right)^{1 / 2}\left(\int_{-\infty}^{\infty}\left(\phi_{t z}^{2}+\phi_{z z}^{2}\right) d y\right)^{1 / 2} \rightarrow 0 \quad \text { as } t \rightarrow \infty
\end{aligned}
$$

which is sufficient to imply (3.11).

The strategy of obtaining the global existence for $\phi$ is the following local existence theorem of $\phi$ combined with an a priori estimate.

Proposition 3.2 (local existence). For any $\varepsilon_{2}>0$, there exists a constant $T_{1}>0$, depending on $\varepsilon_{2}$, such that if $\phi_{0} \in H^{3} \cap H_{w}^{1}$ and $\phi_{1} \in H^{2} \cap L_{w}^{2}$, with $\mathcal{E}(0)<\varepsilon_{2} / 2$, then the problem (3.5), (3.8) has a unique solution $\phi \in X\left(0, T_{1}\right)$ satisfying

$$
\mathcal{E}(t)<2 \mathcal{E}(0)
$$

for any $0 \leq t \leq T_{1}$.

Proposition 3.3 (a priori estimate). Let $\phi \in X(0, T)$ be a solution for a constant $T>0$; then there exists a constant $\varepsilon_{3}>0$, independent of $T$, such that if

$$
\mathcal{E}(t)<\varepsilon_{3} \quad \text { for } t \in[0, T]
$$

then $\phi$ satisfies (3.10) for any $0 \leq t \leq T$.

Note that, since $a$ is sufficiently smooth, the appearance of $a$ does not affect the local existence, so we refer the readers to $[2,6,12,26,29,33,36,39]$ for Proposition 3.2. Therefore, proving Proposition 3.3 becomes the main task in the following section.

\section{Energy estimates}

In this section, we will complete the proof of the stability theorem. The stability result is established by the weighted energy estimates and

(1) the smallness of $\left|a_{+}-a_{-}\right|,\left|\tilde{\rho}_{+}-\tilde{\rho}_{-}\right|$;

(2) entropy condition (1.9);

(3) subsonic condition (1.11);

(4) modified subcharacteristic condition (1.12);

(5) the following properties of traveling wave solutions $(a, U, V)(z)$ (see Lemma 2.2):

(a) monotonicity of $U(z)$;

(b) $\left|U_{z}\right| \geq C\left|a_{z}\right|$ for some constant $C>0$.

Since the traveling wave solutions in all cases (A1)-(B2) satisfy (5a) and (5b), the weighted energy estimates for all cases are similar. So we only provide the proofs for case (A1).

Lemma 4.1. Under the hypothesis given in Theorem 1.1, there exists a constant $C>0$ such that any solution $\phi \in X(0, T)$ of problem (3.3) satisfies

$$
\begin{aligned}
& \|\phi(t)\|_{H_{w}^{1}}^{2}+\left\|\phi_{t}(t)\right\|_{L_{w}^{2}}^{2}+\int_{0}^{t}\left\|\left(\phi_{t}, \phi_{z}\right)(s)\right\|_{L_{w}^{2}}^{2} d s+\int_{0}^{t} \int_{\mathbb{R}}\left|U_{z}\right| \phi^{2} d z d s \\
\leq & C\left\{\left\|\phi_{0}\right\|_{H^{3}}^{2}+\left\|\phi_{1}\right\|_{H^{2}}^{2}+\int_{0}^{t} \int_{\mathbb{R}} w|F|\left(|\phi|+\left|\left(\phi_{t}-s \phi_{z}\right)\right|\right) d z d s\right\}
\end{aligned}
$$


for $t \in[0, T), 0<T \leq \infty$, where $F$ is given in (3.6), (3.7).

Proof. In view of $w=w(a, U)>0$ in (1.14), we multiply (3.5) by $2 w \phi$ and obtain

$$
2 w(a, U) \phi L(a, \phi)=-2 F w(a, U) \phi .
$$

The left hand side of (4.2) can be reduced to

$$
\begin{aligned}
& 2\left\{\left(\phi_{t}-s \phi_{z}\right)_{t}-s\left(\phi_{t}-s \phi_{z}\right)_{z}-\left(g_{\tilde{\rho}} \phi_{z}\right)_{z}+\left(g_{m}\left(\phi_{t}-s \phi_{z}\right)\right)_{z}\right\} w \phi+\frac{2}{\tau} w \phi \phi_{t}+2 w \phi \mu \phi_{z} \\
= & \left\{2 w \phi\left(\phi_{t}-s \phi_{z}\right)+\left(\frac{1}{\tau} w+s w_{z}-w_{z} g_{m}\right) \phi^{2}\right\}_{t}-2 w\left(\phi_{t}-s \phi_{z}\right)^{2}+2 w g_{\tilde{\rho}} \phi_{z}^{2} \\
& \quad-2 w g_{m} \phi_{z}\left(\phi_{t}-s \phi_{z}\right)+A(U) \phi^{2}+2 a_{z}\left\{g_{a \tilde{\rho}} w+w_{a}\left(g_{\tilde{\rho}}+s g_{m}-s^{2}\right)\right\} \phi \phi_{z}+\{\cdots\}_{z},
\end{aligned}
$$

where $\{\cdots\}_{z}$ denotes the terms which will disappear through the integration with respect to $z \in \mathbb{R}$, and

$$
\begin{aligned}
A(U) & =-\left\{\left(g_{\tilde{\rho}}+s g_{m}-s^{2}\right) w_{\tilde{\rho}} U_{z}+\left(\mu-a_{z} g_{a \tilde{\rho}}\right) w\right\}_{z} \\
& =-\left\{\left(\frac{1}{\tau} Q(a, U)+a_{z}\left(P-g_{a}\right)\right) w_{\tilde{\rho}}+\left(\frac{1}{\tau} Q(a, U)+a_{z}\left(P-g_{a}\right)\right)_{\tilde{\rho}} w\right\}_{z} \\
& =-\left\{w\left(\frac{1}{\tau} Q(a, U)+a_{z}\left(P-g_{a}\right)\right)\right\}_{\tilde{\rho} \tilde{\rho}} U_{z} .
\end{aligned}
$$

Note that the second equality in (4.3) holds due to (2.10). Since $U$ is monotone increasing and

$$
\left\{w\left(\frac{1}{\tau} Q(a, U)+a_{z}\left(P-g_{a}\right)\right)\right\}_{\tilde{\rho} \tilde{\rho}}=-2,
$$

we have

$$
A(U)=2 U_{z}>0
$$

Next, we calculate

$$
2\left(\phi_{t}-s \phi_{z}\right) w L(a, \phi)=-2 F\left(\phi_{t}-s \phi_{z}\right) w .
$$

The left hand side of (4.5) is

$$
\begin{aligned}
2\left\{\left(\phi_{t}-\right.\right. & \left.\left.s \phi_{z}\right)_{t}-s\left(\phi_{t}-s \phi_{z}\right)_{z}-\left(g_{\tilde{\rho}} \phi_{z}\right)_{z}+\left(g_{m}\left(\phi_{t}-s \phi_{z}\right)\right)_{z}\right\}\left(\phi_{t}-s \phi_{z}\right) w \\
& +\frac{2}{\tau}\left(f_{\tilde{\rho}} \phi_{z}+\phi_{t}-s \phi_{z}\right) w\left(\phi_{t}-s \phi_{z}\right)+2 a_{z} w P_{\tilde{\rho}} \phi_{z}\left(\phi_{t}-s \phi_{z}\right) \\
=\left\{w \left(\phi_{t}-\right.\right. & \left.\left.s \phi_{z}\right)^{2}+w g_{\tilde{\rho}} \phi_{z}^{2}\right\}_{t}+\left\{\frac{2}{\tau} w+s w_{z}-2 w_{z} g_{m}+\left(w g_{m}\right)_{z}\right\}\left(\phi_{t}-s \phi_{z}\right)^{2} \\
& +s\left(w g_{\tilde{\rho}}\right)_{z} \phi_{z}^{2}+\left\{2 w_{z} g_{\tilde{\rho}}+\frac{2}{\tau} w f_{\tilde{\rho}}+2 w a_{z} P_{\tilde{\rho}}\right\} \phi_{z}\left(\phi_{t}-s \phi_{z}\right)+\{\cdots\}_{z} .
\end{aligned}
$$

Hence, multiplying (4.5) by $2 \tau$ and adding to (4.2), we obtain

$$
\begin{aligned}
& \left\{E_{1}\left(\phi,\left(\phi_{t}-s \phi_{z}\right)\right)+E_{3}\left(\phi_{z}\right)\right\}_{t}+E_{2}\left(\phi_{z},\left(\phi_{t}-s \phi_{z}\right)\right)+E_{4}(\phi) \\
& +2 a_{z}\left\{g_{a \tilde{\rho}} w+w_{a}\left(g_{\tilde{\rho}}+s g_{m}-s^{2}\right)\right\} \phi \phi_{z}+\{\cdots\}_{z} \\
= & -2 F w\left\{\phi+2 \tau\left(\phi_{t}-s \phi_{z}\right)\right\}
\end{aligned}
$$


where

$$
\begin{aligned}
& E_{1}\left(\phi,\left(\phi_{t}-s \phi_{z}\right)\right)=2 \tau w\left(\phi_{t}-s \phi_{z}\right)^{2}+2 w \phi\left(\phi_{t}-s \phi_{z}\right)+\left\{\frac{1}{\tau} w+s w_{z}-w_{z} g_{m}\right\} \phi^{2}, \\
& E_{2}\left(\phi_{z},\left(\phi_{t}-s \phi_{z}\right)\right)=2\{w\left.+\tau s w_{z}-2 \tau w_{z} g_{\tilde{\rho}}+\tau\left(w g_{m}\right)_{z}\right\}\left(\phi_{t}-s \phi_{z}\right)^{2} \\
&+2\left(2 f_{\tilde{\rho}} w+2 \tau w_{z} g_{\tilde{\rho}}-w g_{m}+2 \tau w a_{z} P_{\tilde{\rho}}\right) \phi_{z}\left(\phi_{t}-s \phi_{z}\right) \\
&+2\left\{w g_{\tilde{\rho}}+\tau s\left(w g_{\tilde{\rho}}\right)_{z}\right\} \phi_{z}^{2}, \\
& E_{3}\left(\phi_{z}\right)=2 \tau w g_{\tilde{\rho}} \phi_{z}^{2} \\
& E_{4}(\phi)=A(U) \phi^{2}
\end{aligned}
$$

Here $A(U)$ is defined in (4.3).

The discriminants of the quadratics $E_{j}(j=1,2)$ are respectively

$$
\begin{aligned}
& D_{1}=-4 w\left(w+2 \tau s w_{z}+2 \tau g_{m} w_{z}\right), \\
& \begin{aligned}
D_{2}=4\left(2 f_{\tilde{\rho}} w+2 \tau w_{z} g_{\tilde{\rho}}-w g_{m}+2 \tau w a_{z} p_{\tilde{\rho}}\right)^{2} \\
\quad-16\left\{w+\tau s w_{z}-2 \tau w_{z} g_{m}+\tau\left(w g_{m}\right)_{z}\right\}\left\{w g_{\tilde{\rho}}-\tau s\left(w g_{\tilde{\rho}}\right)_{z}\right\} .
\end{aligned}
\end{aligned}
$$

Now, under (1.11), (1.12), and the condition that $\left|a_{+}-a_{-}\right|,\left|\tilde{\rho}_{+}-\tilde{\rho}_{-}\right|$are sufficiently small, we claim that

$$
D_{1}<0, \quad D_{2}<0 .
$$

Indeed, the inequalities in (4.7) are equivalent to

$$
\begin{gathered}
1+2 \tau s \frac{w_{z}}{w}+2 \tau g_{m} \frac{w_{z}}{w}>0, \\
\left(f_{\tilde{\rho}}-\frac{1}{2} g_{m}+\tau g_{\tilde{\rho}} \frac{w_{z}}{w}+\tau a_{z} P_{\tilde{\rho}}\right)^{2} \\
<\left\{1+\tau s \frac{w_{z}}{w}-2 \tau g_{m} \frac{w_{z}}{w}+\tau \frac{\left(w g_{m}\right)_{z}}{w}\right\}\left\{g_{\tilde{\rho}}-\tau s \frac{\left(w g_{\tilde{\rho}}\right)_{z}}{w}\right\} .
\end{gathered}
$$

Note that $\left|\tau w_{z}\right|,\left|\tau a_{z}\right|,\left|\tau\left(w g_{m}\right)_{z}\right|$, and $\left|\tau\left(w g_{\tilde{\rho}}\right)_{z}\right|$ are small provided that $\left|a_{+}-a_{-}\right|$, $\left|\tilde{\rho}_{+}-\tilde{\rho}_{-}\right|$are sufficiently small. Therefore, by (1.11), (1.12) together with the fact above, we achieve inequalities (4.8) and (4.9), which implies (4.7).

Therefore, by (4.7) there exist constants $M_{0}, M>0$ such that

$$
\left\{\begin{array}{l}
M_{0} w\left\{\phi^{2}+\left(\phi_{t}-s \phi_{z}\right)^{2}\right\} \leq E_{1} \leq M w\left\{\phi^{2}+\left(\phi_{t}-s \phi_{z}\right)^{2}\right\} \\
M_{0} w\left\{\phi_{z}^{2}+\left(\phi_{t}-s \phi_{z}\right)^{2}\right\} \leq E_{2} .
\end{array}\right.
$$

Furthermore, (1.11) and (4.4) yield

$$
\left\{\begin{array}{l}
E_{3}=2 \tau w g_{\tilde{\rho}} \phi_{z}^{2} \geq 0 \\
E_{4}=2 U_{z} \phi^{2} \geq 0
\end{array}\right.
$$

Next, we estimate the fourth term in (4.6). Applying Lemma 2.2, there exists a constant $C_{0}>0$ such that $U_{z} \geq C_{0}\left|a_{z}\right|$. Therefore, by Young's inequality,

$$
\left|2 a_{z}\left\{g_{a \tilde{\rho}} w+w_{a}\left(g_{\tilde{\rho}}+s g_{m}-s^{2}\right)\right\} \phi \phi_{z}\right| \leq C_{0}\left|a_{z}\right| \phi^{2}+M_{0} a_{z} \phi_{z}^{2} \leq U_{z} \phi^{2}+M_{0} a_{z} \phi_{z}^{2},
$$


where $M_{0} \geq C_{0}^{-1}\left|g_{a \tilde{\rho}} w+w_{a}\left(g_{\tilde{\rho}}+s g_{m}-s^{2}\right)\right|^{2}$. Since $\left|a_{z}\right|$ is small if $\left|a_{+}-a_{-}\right|$is small, we conclude that the fourth term in (4.6) is dominated by $E_{2}$ and $E_{4}$.

Substituting estimates (4.10), (4.11), and (4.12) into (4.6) and integrating (4.6) with respect to $t$ and $z$, we arrive at the desired estimate (4.1).

Next, we estimate the higher order derivatives of $\phi$.

Lemma 4.2. Under the hypothesis given in Theorem 1.1, there exists $C>0$ such that any solution $\phi \in X(0, T)$ of problem (3.3) satisfies

$$
\begin{gathered}
\left\|\phi_{z}(t)\right\|_{H^{1}}^{2}+\left\|\phi_{z t}(t)\right\|_{L^{2}}^{2}+\int_{0}^{t}\left\|\left(\phi_{z t}, \phi_{z z}\right)(s)\right\|_{L^{2}}^{2} d s \\
\leq C\left\{\left\|\phi_{0}\right\|_{H^{2}}^{2}+\left\|\phi_{1}\right\|_{H^{1}}^{2}+\int_{0}^{t} \int_{\mathbb{R}}\left|F_{z}\right|\left(\left|\phi_{z}\right|+\left|\left(\phi_{z t}, \phi_{z z}\right)\right|\right) d z d s\right. \\
\left.\quad+\left\|\phi_{0}\right\|_{H_{w}^{1}}^{2}+\left\|\phi_{1}\right\|_{L_{w}^{2}}^{2}+\int_{0}^{t} \int_{\mathbb{R}} w|F|\left(|\phi|+\left|\left(\phi_{t}, \phi_{z}\right)\right|\right) d z d s\right\}
\end{gathered}
$$

for $t \in[0, T), 0<T \leq \infty$, where $F$ is given in (3.6), (3.7).

Proof. Let $\phi_{z}:=\Phi$. Then a direct calculation leads to

$$
\begin{aligned}
\partial_{z} L(a, \phi)=\left(\phi_{z t}-s \phi_{z z}\right)_{t}-s\left(\phi_{z t}-s \phi_{z z}\right)_{z}-\left\{g_{\tilde{\rho}} \phi_{z z}+g_{m}\left(s \phi_{z z}-\phi_{z t}\right)\right\}_{z} \\
+\frac{1}{\tau} \phi_{z t}+\mu \phi_{z z}-\left\{\left(g_{\tilde{\rho}}\right)_{z} \phi_{z}+\left(g_{m}\right)_{z}\left(s \phi_{z}-\phi_{t}\right)\right\}_{z}+\mu_{z} \phi_{z} \\
=L(a, \Phi)-\left\{\left(g_{\tilde{\rho}}\right)_{z} \phi_{z}+\left(g_{m}\right)_{z}\left(s \phi_{z}-\phi_{t}\right)\right\}_{z}+\mu_{z} \phi_{z} .
\end{aligned}
$$

Multiplying $\partial_{z} L(a, \phi)$ by $2 \phi_{z}$ and $2\left(\phi_{t}-s \phi_{z}\right)_{z}$ respectively, we have

$$
\begin{aligned}
2 \partial_{z} L(a, \phi) \phi_{z} & =-2 F_{z} \phi_{z}, \\
2 \partial_{z} L(a, \phi)\left(\phi_{t}-s \phi_{z}\right)_{z} & =-2 F_{z}\left(\phi_{t}-s \phi_{z}\right)_{z} .
\end{aligned}
$$

Next, following the argument in [17, 21], we apply a similar analysis in obtaining the left hand side of (4.2), (4.5) with $w=1$ to get

$$
\begin{aligned}
2 \partial_{z} L(a, \phi) \phi_{z}=\{ & \left.2 \Phi\left(\Phi_{t}-s \Phi_{z}\right)+\left(\frac{1}{\tau}+\left(g_{m}\right)_{z}\right) \Phi^{2}\right\}_{t}-2\left(\Phi_{t}-s \Phi_{z}\right)^{2}+2 g_{\tilde{\rho}} \Phi_{z}^{2} \\
& -2 g_{m} \Phi_{z}\left(\Phi_{t}-s \Phi_{z}\right)+\left\{\mu_{z}+\left(s g_{m}-g_{\tilde{\rho}}\right)_{z z}\right\} \phi_{z}^{2} \\
& +2\left(g_{m}\right)_{z z} \phi_{z}\left(\phi_{t}-s \phi_{z}\right)+\{\cdots\}_{z}
\end{aligned}
$$

and

$$
\begin{aligned}
2 \partial_{z} L(a, \phi)\left(\phi_{t}-s \phi_{z}\right)_{z}=\left\{\left(\Phi_{t}-\right.\right. & \left.\left.s \Phi_{z}\right)^{2}+\left(g_{\tilde{\rho}}-\left(g_{\tilde{\rho}}\right)_{z z}+\mu_{z}\right) \Phi^{2}\right\}_{t}+s\left(g_{\tilde{\rho}}\right)_{z} \Phi_{z}^{2} \\
& +\left\{\frac{2}{\tau} f_{\tilde{\rho}}+2 a_{z} P_{\tilde{\rho}}-2\left(g_{\tilde{\rho}}\right)_{z}\right\} \Phi_{z}\left(\Phi_{t}-s \Phi_{z}\right) \\
& +\left\{\frac{2}{\tau}+3\left(g_{m}\right)_{z}\right\}\left(\Phi_{t}-s \Phi_{z}\right)^{2}-s\left(g_{\tilde{\rho}}\right)_{z z z} \phi_{z}^{2} \\
& -\left(g_{m}\right)_{z z z}\left(\phi_{t}-s \phi_{z}\right)^{2}+s \mu_{z z} \phi_{z}^{2}+\{\cdots\}_{z} .
\end{aligned}
$$

Multiplying (4.15) by $2 \tau$ and adding to (4.14), we obtain

$$
\begin{aligned}
& \left\{E_{1}\left(\Phi,\left(\Phi_{t}-s \Phi_{z}\right)\right)+E_{3}\left(\Phi_{z}\right)\right\}_{t}+E_{2}\left(\Phi_{z},\left(\Phi_{t}-s \Phi_{z}\right)\right)+G\left(\phi_{t}, \phi_{z}\right)+\{\cdots\}_{z} \\
= & -2 F\left\{\Phi+2 \tau\left(\Phi_{t}-s \Phi_{z}\right)\right\},
\end{aligned}
$$


where

$$
\begin{aligned}
& G\left(\phi_{t}, \phi_{z}\right)=\left\{\mu_{z}+\left(s g_{m}-g_{\tilde{\rho}}\right)_{z z}-2 \tau s\left(g_{\tilde{\rho}}\right)_{z z z}+2 \tau s \mu_{z z}\right\} \phi_{z}^{2} \\
& +2\left(g_{m}\right)_{z z} \phi_{z}\left(\phi_{t}-s \phi_{z}\right)-2 \tau\left(g_{m}\right)_{z z z}\left(\phi_{t}-s \phi_{z}\right)^{2}, \\
& E_{1}\left(\Phi,\left(\Phi_{t}-s \Phi_{z}\right)\right)=2 \tau\left(\Phi_{t}-s \Phi_{z}\right)^{2}+2 \Phi\left(\Phi_{t}-s \Phi_{z}\right) \\
& +\left\{\frac{1}{\tau}+\left(g_{m}\right)_{z}-2 \tau\left(g_{\tilde{\rho}}\right)_{z z}+2 \tau \mu_{z}\right\} \Phi^{2}, \\
& E_{2}\left(\Phi_{z},\left(\Phi_{t}-s \Phi_{z}\right)\right)=\left\{2+6 \tau\left(g_{m}\right)_{z}\right\}\left(\Phi_{t}-s \Phi_{z}\right)^{2}+\left\{2 g_{\tilde{\rho}}+2 \tau s\left(g_{\tilde{\rho}}\right)_{z}\right\} \Phi_{z}^{2} \\
& +2\left\{2 f_{\tilde{\rho}}-2 \tau\left(g_{\tilde{\rho}}\right)_{z}-g_{m}+2 \tau a_{z} P_{\tilde{\rho}}\right\} \Phi_{z}\left(\Phi_{t}-s \Phi_{z}\right), \\
& E_{3}\left(\Phi_{z}\right)=2 \tau g_{\tilde{\rho}} \Phi_{z}^{2} .
\end{aligned}
$$

The discriminants of the quadratics $E_{j}(j=1,2)$ are respectively

$$
\begin{aligned}
D_{1} & =4-8 \tau\left\{\frac{1}{\tau}+\left(g_{m}\right)_{z}-2 \tau\left(g_{\tilde{\rho}}\right)_{z z}+2 \tau \mu_{z}\right\} \\
& =4-8 \tau\left\{\frac{1}{\tau}+\left(g_{m}\right)_{z}-2 \tau\left(g_{\tilde{\rho}}\right)_{z z}+2\left(f_{\tilde{\rho}}\right)_{z}+2 \tau\left(a_{z} P_{\tilde{\rho}}\right)_{z}\right\}, \\
D_{2} & =4\left\{2 f_{\tilde{\rho}}-2 \tau\left(g_{\tilde{\rho}}\right)_{z}-g_{m}+2 \tau a_{z} P_{\tilde{\rho}}\right\}^{2}-4\left\{2+6 \tau\left(g_{m}\right)_{z}\right\}\left\{2 g_{\tilde{\rho}}+2 \tau s\left(g_{\tilde{\rho}}\right)_{z}\right\} .
\end{aligned}
$$

Following a similar argument as in the proof of Lemma 4.1, we arrive at

$$
D_{1}<0 \text { and } D_{2}<0 \text {, }
$$

provided that $\left|a_{+}-a_{-}\right|,\left|\tilde{\rho}_{+}-\tilde{\rho}_{-}\right|$are sufficiently small and the conditions (1.11), (1.12) hold.

Now, integrating (4.16) with respect to $t$ and $z$, we have the following estimate:

$$
\begin{aligned}
& \|\Phi(t)\|_{H^{1}}^{2}+\left\|\Phi_{t}(t)\right\|_{L^{2}}^{2}+\int_{0}^{t}\left\|\left(\Phi_{t}, \Phi_{z}\right)(s)\right\|_{L^{2}}^{2} d s \\
\leq & C\left\{\left\|\phi_{0}^{\prime}\right\|_{H^{1}}^{2}+\left\|\phi_{1}^{\prime}\right\|_{L^{2}}^{2}+\int_{0}^{t} \int_{\mathbb{R}}\left|G\left(\phi_{t}, \phi_{z}\right)\right| d z d s+\int_{0}^{t} \int_{\mathbb{R}}\left|F_{z}\right|\left(\Phi+\left(\Phi_{t}, \Phi_{z}\right) \mid\right) d z d s\right\},
\end{aligned}
$$

where $C>0$ is a constant. Furthermore, using the estimate (4.1), we obtain

$$
\begin{aligned}
\int_{0}^{t} \int_{\mathbb{R}}\left|G\left(\phi_{t}, \phi_{z}\right)\right| d z d s & \leq C \int_{0}^{t}\left\|\left(\phi_{t}, \phi_{z}\right)(s)\right\|_{L^{2}}^{2} d s \\
& \leq C\left\{\left\|\phi_{0}\right\|_{H_{w}^{1}}^{2}+\left\|\phi_{1}\right\|_{L_{w}^{2}}^{2}+\int_{0}^{t} \int_{\mathbb{R}} w|F|\left(|\phi|+\left|\left(\phi_{t}, \phi_{z}\right)\right|\right) d z d s\right\} .
\end{aligned}
$$
(4.13).

Finally, substituting (4.18) into (4.17) and replacing $\Phi$ by $\phi_{z}$, we get the inequality

Next, (3.5) yields

$$
2 \partial_{z}^{2} L(a, \phi) \phi_{z z}+4 \tau \partial_{z}^{2} L(a, \phi)\left(\phi_{t}-s \phi_{z}\right)_{z z}=-2 F_{z z}\left\{\phi_{z z}+2 \tau\left(\phi_{t}-s \phi_{z}\right)_{z z}\right\} .
$$

Setting $\Psi=\phi_{z z}$, we have

$$
\begin{aligned}
\partial_{z}^{2} L(a, \phi)=L(a, \Psi)+\left\{g_{m}\left(\phi_{t}-s \phi_{z}\right)-g_{\tilde{\rho}} \phi_{z}\right\}_{z z z} \\
-\left\{g_{m}\left(\Psi_{t}-s \Psi_{z}\right)-g_{\tilde{\rho}} \Psi_{z}\right\}_{z}+(\mu \Phi)_{z z}-\mu \Psi_{z} .
\end{aligned}
$$


Then, a straightforward calculation gives

$$
\begin{aligned}
2 \partial_{z}^{2} L(a, \phi) \phi_{z z}=\{ & \left.2 \Psi\left(\Psi_{t}-s \Psi_{z}\right)+\frac{1}{\tau} \Psi^{2}\right\}_{t}-2\left(\Psi_{t}-s \Psi_{z}\right)^{2}+2 g_{\tilde{\rho}} \Psi_{z}^{2}-2 g_{m} \Psi_{z}\left(\Psi_{t}-s \Psi_{z}\right) \\
& +2 \mu_{z z} \phi_{z} \Psi+3 \mu_{z} \Psi^{2}+\{\cdots\}_{z}-J_{1}
\end{aligned}
$$

and

$$
\begin{aligned}
2 \partial_{z}^{2} L(a, \phi)\left(\phi_{t}-s \phi_{z}\right)_{z z}=\{ & \left.\left(\Psi_{t}-s \Psi_{z}\right)^{2}+g_{\tilde{\rho}} \Psi_{z}^{2}\right\}_{t}+\left\{\left(g_{m}\right)_{z}+\frac{2}{\tau}\right\}\left(\Psi_{t}-s \Psi_{z}\right)^{2}+s\left(g_{\tilde{\rho}}\right)_{z} \Psi_{z}^{2} \\
& +\left\{\frac{2}{\tau} f_{\tilde{\rho}}+2 a_{z} P_{\tilde{\rho}}\right\} \Psi_{z}\left(\Psi_{t}-s \Psi_{z}\right)+2 \mu_{z z} \phi_{z}\left(\Psi_{t}-s \Psi_{z}\right) \\
& +4 \mu_{z} \Psi\left(\Psi_{t}-s \Psi_{z}\right)+\{\cdots\}_{z}-J_{2}
\end{aligned}
$$

where

$$
\begin{aligned}
& J_{1}=2 \Psi\left\{\left(g_{\tilde{\rho}} \phi_{z}-g_{m}\left(\phi_{t}-s \phi_{z}\right)\right)_{z z z}-\left(g_{\tilde{\rho}} \Psi_{z}-g_{m}\left(\Psi_{t}-s \Psi_{z}\right)\right)_{z}\right\} \\
& J_{2}=2\left(\Psi_{t}-s \Psi_{z}\right)\left\{\left(g_{\tilde{\rho}} \phi_{z}-g_{m}\left(\phi_{t}-s \phi_{z}\right)\right)_{z z z}-\left(g_{\tilde{\rho}} \Psi_{z}-g_{m}\left(\Psi_{t}-s \Psi_{z}\right)\right)_{z}\right\}
\end{aligned}
$$

It follows from (4.19) that

$$
\begin{gathered}
\left\{2 \tau\left(\Psi_{t}-s \Psi_{z}\right)^{2}+2 \Psi\left(\Psi_{t}-s \Psi_{z}\right)+\frac{1}{\tau} \Psi^{2}+2 \tau g_{\tilde{\rho}} \Psi_{z}^{2}\right\}_{t}+\left\{2 g_{\tilde{\rho}}+2 \tau s\left(g_{\tilde{\rho}}\right)_{z}\right\} \Psi_{z}^{2} \\
+\left\{2+2 \tau\left(g_{m}\right)_{z}\right\}\left(\Psi_{t}-s \Psi_{z}\right)^{2}+\left\{4 f_{\tilde{\rho}}-2 g_{m}+4 \tau a_{z} P_{\tilde{\rho}}\right\} \Psi_{z}\left(\Psi_{t}-s \Psi_{z}\right) \\
+8 \tau \mu_{z} \Psi\left(\Psi_{t}-s \Psi_{z}\right)+3 \mu_{z} \Psi^{2}+2 \mu_{z z} \phi_{z} \Psi+4 \tau \mu_{z z} \phi_{z}\left(\Psi_{t}-s \Psi_{z}\right)+\{\cdots\}_{z} \\
=-2 F_{z z}\left\{\phi_{z z}+2 \tau\left(\phi_{t}-s \phi_{z}\right)_{z z}\right\}+J,
\end{gathered}
$$

where $J:=J_{1}+J_{2}$. Since $\left|a_{+}-a_{-}\right|,\left|\tilde{\rho}_{+}-\tilde{\rho}_{-}\right| \ll 1$,

$$
\begin{aligned}
|J| & =2\left|\left\{\Psi+2 \tau\left(\Psi_{t}+s \Phi_{z}\right)\right\}\left\{\left(g_{\tilde{\rho}} \phi_{z}-g_{m}\left(\phi_{t}-s \phi_{z}\right)\right)_{z z z}-\left(g_{\tilde{\rho}} \Psi_{z}-g_{m}\left(\Psi_{t}-s \Psi_{z}\right)\right)_{z}\right\}\right| \\
& \leq \frac{1}{3}\left|\left(\Psi_{t}, \Psi_{z}\right)\right|^{2}+C\left|\left(\phi_{t}, \phi_{z}, \phi_{z t}, \phi_{z z}\right)\right|^{2} .
\end{aligned}
$$

We observe that $\mu_{z}, \mu_{z z}$ are smooth bounded functions. Thus, noting that $\Psi=\phi_{z z}$ and applying Young's inequality for the mixed terms $8 \tau \mu_{z} \Psi\left(\Psi_{t}-s \Psi_{z}\right)$ and $4 \tau \mu_{z z} \phi_{z}\left(\Psi_{t}-\right.$ $\left.s \Psi_{z}\right)$ in (4.20), we obtain the following lemma.

Lemma 4.3. Under the hypothesis given in Theorem 1.1, there exists $C>0$ such that any solution $\phi \in X(0, T)$ of problem (3.3) satisfies

$$
\begin{aligned}
& \left\|\phi_{z z}(t)\right\|_{H^{1}}^{2}+\left\|\phi_{z z t}(t)\right\|_{L^{2}}^{2}+\frac{1}{3} \int_{0}^{t}\left\|\left(\phi_{z z t}, \phi_{z z z}\right)(s)\right\|_{L^{2}}^{2} d s-C \int_{0}^{t}\left\|\left(\phi_{t}, \phi_{z}\right)\right\|_{H^{1}}^{2} d s \\
\leq & C\left\{\left\|\phi_{0}\right\|_{H^{3}}^{2}+\left\|\phi_{1}\right\|_{H^{2}}^{2}+\left|\int_{0}^{t} \int_{\mathbb{R}} F_{z z}\left\{\phi_{z z}+2 \tau\left(\phi_{t}-s \phi_{z}\right)_{z z}\right\} d z d s\right|\right\}
\end{aligned}
$$

for $t \in[0, T), 0<T \leq \infty$, where $F$ is given in (3.6), (3.7).

Combining estimates (4.1), (4.13), and (4.21), we finally obtain

$$
\begin{gathered}
\|\phi(t)\|_{H^{3}}^{2}+\left\|\phi_{t}(t)\right\|_{H^{2}}^{2}+\left\|\phi_{t}(t)\right\|_{H_{w}^{1}}^{2}+\left\|\phi_{t}(t)\right\|_{L_{w}^{2}}^{2}+\int_{0}^{t} \int_{\mathbb{R}}\left|U_{z}\right| \phi^{2} d z d s \\
+\int_{0}^{t}\left\|\left(\phi_{t}, \phi_{z}\right)\right\|_{H^{2}}^{2} d s+\int_{0}^{t}\left\|\left(\phi_{t}, \phi_{z}\right)\right\|_{L_{w}^{2}}^{2} d s
\end{gathered}
$$




$$
\begin{aligned}
\leq C\left\{\left\|\phi_{0}\right\|_{H^{3}}^{2}+\left\|\phi_{1}\right\|_{H^{2}}^{2}+\left\|\phi_{0}\right\|_{H_{w}^{1}}^{2}+\left\|\phi_{1}\right\|_{L_{w}^{2}}^{2}+\int_{0}^{t} \int_{\mathbb{R}} w|F|\left(|\phi|+\left|\left(\phi_{t}, \phi_{z}\right)\right|\right) d z d s\right. \\
+\int_{0}^{t} \int_{\mathbb{R}}\left|F_{z}\right|\left(\left|\phi_{z}\right|+\left|\left(\phi_{z t}, \phi_{z z}\right)\right|\right) d z d s \\
\left.+\left|\int_{0}^{t} \int_{\mathbb{R}} F_{z z}\left\{\phi_{z z}+2\left(\phi_{t}-s \phi_{z}\right)_{z z}\right\} d z d s\right|\right\},
\end{aligned}
$$

where $F$ is given in (3.6), (3.7).

By the definition of $F$ and Taylor's theorem, we have

$$
\begin{aligned}
|F|=O(1)\left(\phi_{t}^{2}+\phi_{z}^{2}+\phi_{z t}^{2}+\phi_{z z}^{2}\right) & \\
\left|F_{z}\right|=O(1)\left(\phi_{t}^{2}+\right. & \left.\phi_{z}^{2}+\phi_{z t}^{2}+\phi_{z z}^{2}+\left|\phi_{z} \phi_{z z z}\right|+\left|\phi_{t} \phi_{z z z}\right|+\left|\phi_{z} \phi_{z z t}\right|+\left|\phi_{t} \phi_{z z t}\right|\right) \\
\left|F_{z z}\right|=O(1)\left(\phi_{t}^{2}+\right. & \phi_{z}^{2}+\phi_{z t}^{2}+\phi_{z z}^{2}+\left|\phi_{z} \phi_{z z z}\right|+\left|\phi_{t} \phi_{z z z}\right|+\left|\phi_{z} \phi_{z z t}\right| \\
& +\left|\phi_{t} \phi_{z z t}\right|+\left|\phi_{z z} \phi_{z z z}\right|+\left|\phi_{t} \phi_{z z z}\right|+\left|\phi_{z z} \phi_{z z t}\right|+\left|\phi_{z t} \phi_{z z t}\right| \\
& \left.+\left|\phi_{z} \phi_{z z z z}\right|+\left|\phi_{t} \phi_{z z z z}\right|+\left|\phi_{z} \phi_{z z z t}\right|+\left|\phi_{t} \phi_{z z z t}\right|\right)
\end{aligned}
$$

Owing to the nonlinearity of function $g$, there are terms containing fourth order derivatives of $\phi$ in $F_{z z}$ which appear in the last term on the right hand side of (4.22). To complete the energy estimate, we apply the integration by parts to reduce the order of derivatives for those terms. For instance, we estimate one of the terms in $\left|\int_{0}^{t} \int_{\mathbb{R}} F_{z z}\left\{\phi_{z z}+2\left(\phi_{t}-s \phi_{z}\right)_{z z}\right\} d z d s\right|:$

$$
\begin{aligned}
& \int_{0}^{t} \int 2 H\left(a, U, V, \phi_{z}, \phi_{t}\right) \phi_{z} \phi_{z z z t} \phi_{z z t} d z d s \\
= & \int_{0}^{t} \int H\left(a, U, V, \phi_{z}, \phi_{t}\right) \phi_{z}\left(\phi_{z z t}^{2}\right)_{z} d z d s \\
= & \int_{0}^{t} \int\left\{\left(H\left(a, U, V, \phi_{z}, \phi_{t}\right) \phi_{z} \phi_{z z t}^{2}\right)_{z}-\left(H\left(a, U, V, \phi_{z}, \phi_{t}\right) \phi_{z}\right)_{z} \phi_{z z t}^{2}\right\} d z d s \\
= & -\int_{0}^{t} \int\left(H\left(a, U, V, \phi_{z}, \phi_{t}\right) \phi_{z}\right)_{z} \phi_{z z t}^{2} d z d s,
\end{aligned}
$$

where

$$
H\left(a, U, V, \phi_{z}, \phi_{t}\right)=g_{m}\left(a, U+\phi_{z}, V-\left(\phi_{t}-s \phi_{z}\right)\right)-g_{m}(a, U, V) .
$$

The estimates for the rest of the terms in $\left|\int_{0}^{t} \int_{\mathbb{R}} F_{z z}\left\{\phi_{z z}+2\left(\phi_{t}-s \phi_{z}\right)_{z z}\right\} d z d s\right|$ involving fourth order derivatives are similar, so we omit them. Therefore, the energy estimates are closed at the derivatives up to third order. Thus in view of (3.9), the integrals on the right hand side of (4.22) are dominated by

$$
C \mathcal{E}(t)\left\{\int_{0}^{t}\left\|\left(\phi_{t}, \phi_{z}\right)\right\|_{H^{2}}^{2} d s+\int_{0}^{t}\left\|\left(\phi_{t}, \phi_{z}\right)\right\|_{L_{w}^{2}}^{2} d s\right\},
$$

which implies that

$$
\begin{aligned}
& \mathcal{E}^{2}(t)+\int_{0}^{t}\left\|\left(\phi_{t}, \phi_{z}\right)\right\|_{H^{2}}^{2} d s+\int_{0}^{t}\left\|\left(\phi_{t}, \phi_{z}\right)\right\|_{L_{w}^{2}}^{2} d s \\
\leq & C \mathcal{E}^{2}(0)+C \mathcal{E}(t)\left\{\int_{0}^{t}\left\|\left(\phi_{t}, \phi_{z}\right)\right\|_{H^{2}}^{2} d s+\int_{0}^{t}\left\|\left(\phi_{t}, \phi_{z}\right)\right\|_{L_{w}^{2}}^{2} d s\right\} .
\end{aligned}
$$


Therefore, by assuming $\mathcal{E}(t) \leq \frac{1}{2 C}$, we establish the desired estimate $\mathcal{E}^{2}(t)+\int_{0}^{t}\left\|\left(\phi_{t}, \phi_{z}\right)\right\|_{H^{2}}^{2} d s+\int_{0}^{t}\left\|\left(\phi_{t}, \phi_{z}\right)\right\|_{L_{w}^{2}}^{2} d s \leq C \mathcal{E}^{2}(0), \quad$ for $t \in[0, T), 0<T \leq \infty$.

Thus, the proof of Proposition 3.3 is completed.

\section{Conclusions}

For convenience, throughout these conclusions, the term "the total amount of the traffic" means the amount of the traffic (or the value of total mass) in a fixed finite interval corresponding to the moving coordinate $z$. Based on our results, the total amount of the traffic is limited in some range when the traffic density forms a traveling wave. The relation between the road widths and the densities at $x= \pm \infty$ controls the behavior of the traveling waves (see (2.14)-(2.17)). That is the reason why we separate cases (A) and (B) into two subcases (A1), (A2) and (B1), (B2) respectively. In cases (A2), (B1), and (B2), the uniqueness of the traveling wave profile in Theorem 1.1 indicates that the density performs as a traveling wave only when the total amount of the traffic attains a specific value. As soon as the total amount changes, the stability of the traveling wave breaks down. However, in case (A1), the traveling waves occur as the total amount of the traffic lies in a proper range. Therefore, the stability will not change under a small perturbation that changes the total amount of traffic. Instead, the traffic density will approach another traveling wave as $t \rightarrow \infty$ (see the first and the second paragraphs in Section 3).

\section{REFERENCES}

[1] G.Q. Chen, C.D. Levermore, and T.P. Liu, Hyperbolic conservation laws with stiff relaxation terms and entropy, Commun. Pure Appl. Math., 47, 787-830, 1993.

[2] P. Dionne, Sur les problèmes de Cauchy hyperboliques bien posés, J. d'Analyse Math., 10, 1-90, 1962.

[3] J. Goodman, Nonlinear asymptotic stability of viscous shock profiles for conservation laws, Arch. Rat. Mech. Anal., 95, 325-344, 1986.

[4] D. Helbing, Traffic and related self-driven many-particle systems, Rev. Modern Phy., 73, 1067$1141,2001$.

[5] J.M. Hong, C.-H. Hsu, B.-C. Huang, and T.-S. Yang, Geometric singular perturbation approach to the existence and instability of stationary waves for viscous traffic flow models, Commun. Pur. Appl. Anal., 12, 1501-1526, 2013.

[6] T.J.R. Hughes, T. Kato, and J.E. Marsden, Well-posed quasi-linear second-order hyperbolic systems with applications to nonlinear elastodynamics and general relativity, Arch. Rat. Mech. Anal., 63, 273-294, 1976.

[7] S.B. Hsu, Ordinary Differential Equations with Applications, World Scientific, 2006.

[8] S. Jin and Z. Xin, The relaxation schemes for systems of conservation laws in arbitrary space dimensions, Commun. Pure Appl. Math., 48, 555-563, 1995.

[9] W.L. Jin and H.M. Zhang, The formation and structure of vehicle clusters in the PayneWhitham traffic flow model, Transport. Res., B., 37, 207-223, 2003.

[10] S. Jin and M.A. Katsoulakis, Hyperbolic systems with supercharacteristic relaxations and roll waves, SIAM J. Appl. Math., 61, 273-292, 2000.

[11] C. Jones, R. Gardner, and T. Kapitula, Stability of traveling waves for non-convex scalar viscous conservation laws, Commun. Pure Appl. Math., 46, 505-526, 1993.

[12] T. Kato, The Cauchy problem for quasi-linear symmetric hyperbolic systems, Arch. Rat. Mech. Anal., 58, 181-205, 1975.

[13] W. Knospe, L. Santen, A. Schadschneider, and M. Schreckenberg, Single-vehicle data of highway traffic: Microscopic description of traffic phases, Phys. Rev. E, 65, 056133, 1-16, 2002.

[14] C. Lattanzio and P. Marcati, The zero relaxation limit for the hydrodynamic Whitham traffic flow model, J. Diff. Equ., 141, 150-178, 1997. 
[15] T. Li, Global solutions of nonconcave hyperbolic conservation laws with relaxation arising from traffic flow, J. Diff. Equ., 190, 131-149, 2003.

[16] T. Li, Nonlinear dynamics of traffic jams, Phys. D, 207, 41-51, 2005.

[17] T. Li, Stability of traveling waves in quasi-linear hyperbolic systems with relaxation and diffusion, SIAM J. Math. Anal., 40, 1058-1075, 2008.

[18] T.P. Liu, Hyperbolic conservation laws with relaxation, Commun. Math. Phys., 108, 153-175, 1987.

[19] H.L. Liu, Asymptotic stability of shock profiles for nonconvex convection-diffusion equation, Appl. Math. Lett., 10, 129-134, 1997.

[20] H.L. Liu, Relaxation Dynamics, Scaling Limits and Convergence of Relaxation Schemes, Analysis and Numerics for Conservation Laws, Springer, Berlin, 453-478, 2005.

[21] T. Li and H.L. Liu, Stability of a traffic flow model with nonconvex relaxation, Commun. Math. Sci., 3, 101-118, 2005.

[22] H.L. Liu and J. Wang, Asymptotic stability of traveling wave solutions of a hyperbolic system with relaxation terms, Beijing Math., 2, 119-130, 1996.

[23] M.J. Lighthill and G.B. Whitham, On kinematic waves: II. A theory of traffic flow on long crowded roads, Proc. Royal Soc. A, 229, 317-345, 1955.

[24] H.L. Liu, C.W. Woo, and T. Yang, Decay rate for traveling waves of a relaxation model, J. Diff. Equ., 134, 343-367, 1997.

[25] H.L. Liu, J. Wang, and T. Yang, Stability for a relaxation model with a nonconvex flux, SIAM J. Math. Anal., 29, 18-29, 1998.

[26] A. Majda, Compressible Fluid Flow and Systems of Conservation Laws in Several Space Variables, Appl. Math. Sci., Springer-Verlag, New York, 53, 1984.

[27] A. Matsumura and K. Nishihara, Asymptotic stability of traveling waves of scalar viscous conservation laws with non-convex nonlinearity, Commun. Math. Phys., 165, 83-96, 1994.

[28] M. Mei, Stability of shock profiles for non-convex scalar conservation laws, Math. Models Meth. Appl. Sci., 5, 279-296, 1995.

[29] T. Nishida, Nonlinear Hyperbolic Equations and Related Topics in Fluid Dynamics, Publ. Math. d'Orsay, 78-02, Départment de Mathématique, Université Paris-sud, Orsay, 1978.

[30] H.J. Payne, Models of freeway traffic and control, Simulation Councils Proceedings Series: Mathematical Models of Public Systems, G.A. Bekey (Eds.), 1, 51-61, 1971.

[31] P.I. Richards, Shock waves on the highway, Op. Res., 4, 42-51, 1956.

[32] D. Sattinger, On the stability of waves of nonlinear parabolic systems, Adv. Math., 22, 312-355, 1976.

[33] J. Schauder, Das anfangswertproblem einer quasilinearen hyperbolischen differentialgleichung zweiter ordnung in beliebiger anzahl von unabhängigen veran̈derlichen, Fund. Math., 24, 213-246, 1935.

[34] S. Schochet, The instant-response limit in Whitham's nonlinear traffic-flow model: uniform well-posedness and global existence, Asymp. Anal., 1, 263-282, 1988.

[35] J. Smoller, Shock Waves and Reaction-Diffusion Equations, Grundlehren der Mathematischen Wissenschaften, Springer-Verlag, New York, 258, 1994.

[36] S.L. Sobolev, Sur les Équations aux Dérivées Partielles Hyperboliques Non-linéaires, Edizioni Cremonese, Rome, 1961.

[37] T.Q. Tang, C.Y. Li, H.J. Huang, and H.Y. Shang, Macro modeling and analysis of traffic flow with road width, J. Cent. South Univ. Tech., 18, 1757-1764, 2011.

[38] G.B. Whitham, Linear and Nonlinear Waves, Wiley, New York, 1974.

[39] Y. Yamada, Quasilinear wave equations and related nonlinear evolution equations, Nagoya Math. J., 84, 31-83, 1981.

[40] H.M. Zhang, A theory of nonequilibrium traffic flow, Transport. Res. Part B, 32, 485-498, 1998.

[41] H.M. Zhang, Driver memory, traffic viscosity and a viscous vehicular traffic flow model, Transport. Res. Part B, 37, 27-41, 2003.

[42] C.J. Zhu, Asymptotic behavior of solutions for p-system with relaxation, J. Diff. Equ., 180, 273-306, 2002. 